\title{
THE GREAT RECESSION: US DYNAMICS AND SPILLOVERS TO THE WORLD ECONOMY
}

FABIO C. BAGLIANO

CLAUDIO MORANA

Working paper No. 17 - November 2010 


\title{
The Great Recession: US dynamics and spillovers to the world economy
}

\author{
Fabio C. Bagliano* \\ Dipartimento di Scienze Economiche e Finanziarie, Università di Torino (Italy), \\ CeRP-Collegio Carlo Alberto (Moncalieri, Italy) \\ Claudio Morana ${ }^{\dagger}$ \\ Dipartimento di Scienze Economiche e Metodi Quantitativi \\ Università del Piemonte Orientale (Novara, Italy), \\ International Centre for Economic Research (ICER, Torino), \\ CeRP-Collegio Carlo Alberto (Moncalieri, Italy)
}

November $2010^{\ddagger}$

*Electronic address: fabio.bagliano@unito.it

${ }^{\dagger}$ Electronic address: claudio.morana@eco.unipmn.it.

${ }^{\ddagger}$ Acknowledgements. The paper benefited from comments by participants at the 2010 QASS Conference on Macro and Financial Economics (London, May 2010), the International Conference on the Economic Crisis and the Process of European Integration (European Parliament, Brussels, June 2010), the 42nd Annual Conference of the Money, Macro and Finance Research Group (Cyprus, September 2010), and at the 6th Eurostat Colloquium on Modern Tools for Business Cycle Analysis: The Lessons from Global Economic Crisis (Luxembourg, September 2010). Many thanks especially to C. Calomiris and H. Pesaran for many insightful comments and suggestions. 


\begin{abstract}
The paper aims at assessing the mechanics of the Great Recession, considering both its domestic propagation within the US, as well as its spillovers to advanced and emerging economies. A total of 50 countries has been investigated by means of a large-scale open economy macroeconometric model, providing an accurate assessment of the international macro/finance interface over the whole 1980-2009 period. It is found that a boom-bust credit cycle interpretation of the crisis is consistent with the empirical evidence. Moreover, concerning the real effects of the crisis within the US, stronger evidence of an asset prices channel, rather than a liquidity channel, has been detected. The results also support the effectiveness of the expansionary fiscal/monetary policy mix implemented by the Fed and the US government. Concerning the spillovers to the world economy, it is found that while the financial shock has spilled over to foreign countries through US housing and stock price dynamics, as well as excess liquidity creation, the trade channel likely is the key trasmission mechanism of the real shock.
\end{abstract}

Keywords: Great Recession, financial crisis, economic crisis, boombust, credit cycle, international business cycle, factor vector autoregressive models.

JEL classification: C22; E32; F36 


\section{Introduction}

Looking at US business cycle history, various severe recession episodes seem relevant to a complete understanding of recent US macroeconomic and financial developments, in particular the 1929-1933 Great Depression and the Savings and Loans (S\&L) crisis of the 1990s. In fact, though the epicenter of the current financial crisis, i.e. the US subprime mortgage market, is peculiar, some similarities with previous episodes may be noticed.

Likewise the Great Depression and the S\&L crisis, a boom-bust cycle in credit volumes and house and stock prices, fostered by procyclical bank loans, well summarizes the key ingredients of the crisis. ${ }^{1}$ Moreover, likewise in the S\&L episode, both a benign price stability environment and deregulated financial markets worked as amplifying mechanisms. ${ }^{2}$ Indeed, following the 2000 stock market crash and 2001 recession, monetary policy was extremely accommodative, while the deepening of the "originate to distribute" banking model and financial engineering allowed for over stretching of credit. In addition, since late 1990s, large capital inflows were also financing a growing current account deficit in the US, mirrored by a specular surplus in emerging Asian economies. Asset prices misalignments, particularly in the housing and stock markets, then built up as a consequence of the savings-corporate investment imbalance: increasingly risky investments were underwritten and bad loans generated, sowing the seeds of the following bust phase. Still similar to the S\&L crisis, the setting in of the bust phase followed expected, yet not materialized, housing price appreciations, which caused the predatory lending mechanism to break down, leading to a generalized decline in asset prices and tight credit conditions.

From an US domestic phenomenon, the crisis has then quickly spread to the other industrialized countries, due to the tight linkages that the process of securitization and reinsurance in the derivatives market created across major financial institutions worldwide, and, more in general, to the strong degree of international financial and economic integration, triggering local credit crunches and consequent economic crises. Second round effects, albeit delayed, can also be found for emerging economies, particularly for those

\footnotetext{
${ }^{1}$ See Bernanke (1983) and Eichengreen and Mitchener (2004) for a boom-bust interpretation of the Great Depression and the 1990 S\&L crisis. See also Almunia et al. (2009), Bordo and James (2009), Bordo and Landon-Lane (2010), Grossman and Meissner (2010) and Temin (2010) for insightful comparisons of the recent crisis with the Great Depression. An extensive analysis of financial crises in a long-run historical perspective is provided by Reinhart and Rogoff (2009b).

${ }^{2}$ See Levine (2010) for an insightful account of the contribution of financial deregulation and policies, by creating incentives for excessive risk taking, in paving the way to the crisis since the mid 1990s.
} 
more heavily relying on external financing. Despite the expansionary policy interventions implemented worldwide, the effects of the crisis have been quite severe: year-on-year GDP contraction at mid-2009 has indeed been sizable for most OECD member countries, being close to or over $4 \%{ }^{3}$

Against this background, the paper aims at assessing the mechanics of the crisis, i.e. the domestic propagation in the US and its spillover to the other OECD countries, as well as to major emerging economies by means of a large-scale open economy macroeconometric model, composed of near 300 equations and covering a total of 50 countries, set in the factor vector autoregressive (F-VAR) framework. Relative to previous work in the literature, the current paper innovates as to the depth and wideness of the analysis and econometric methodology, providing an accurate investigation of the macro/finance interface within the US and between the US and the world economy. The analysis is carried out on quarterly data for the period 1980:1-2009:1. While the current recessionary episode is expected to be much deeper than any other occurred since the Great Depression, the selected sample is however large enough to cover meaningful previous boom-bust credit cycle episodes, as for instance the US S\&L crisis. To be able to evaluate the boom-bust view of recent crisis episodes and current economic and financial developments, several variables capturing excess liquidity conditions and financial fragility are included in the estimated model.

To preview, the main conclusions of the paper are the following. First, concerning dynamics within the US, our findings are quite consistent with a boom-bust credit cycle interpretation of the crisis, as there is evidence that buoyant US housing and stock markets, as well as low real interest rates over the boom phase of the cycle might have been driven by excessively generous liquidity in the system. The large US trade deficit also likely contributed to the latter dynamics, as huge capital inflows were redirected from the Treasury and stocks markets to the housing market. Finally, there is evidence in favour of the effectiveness of the expansionary fiscal/monetary policy mix implemented by the Fed and the US Government. Second, concerning the spillovers of the crisis to foreign advanced and emerging economies, it is found that the trade channel is the key transmission mechanism of the US economic

\footnotetext{
${ }^{3}$ According to Claessens et al. (2009), severe recessions tend to be deeper and longer lasting than average recessions ( $-5 \%$, rather than $-2 \%$ GDP contraction; lasting 5 quarters rather than 4 quarters), involving house prices contractions, and recovery to pre-recession credit growth rates and upswing in house prices requiring, as for corporate investment, about three years. According to Barro and Ursua (2009), depressions (-10\% GDP growth or less) cum stock market crash ( $-25 \%$ or less) would tend to last about 4 years, with stock prices leading the recovery of about 1 year. See also Reinhart and Rogoff (2009a,b) for additional details.
} 
crisis to the rest of the world, while US housing and stock price dynamics, as well as excess liquidity generation, are the key mechanisms whereby the US financial crisis may have spilled over to foreign countries.

The rest of the paper is organized as follows. In the next section the econometric methodology is introduced, while in section 3 the data and their properties are presented. Then, in section 4 issues on specification and estimation of the F-VAR model are discussed, while in sections 5 and 6 the dynamics of the crisis in the US and its spillovers to foreign countries, respectively, are investigated. Finally, conclusions are drawn in section 7 .

\section{Econometric methodology}

To investigate the linkages among the US and a large number of OECD and emerging economies for a set of key macroeconomic variables, the factor vector autoregressive (F-VAR) framework, derived from a dynamic factor model as in Stock and Watson (2005), is adopted. In this context, observed comovements in the series under study are attributed to a (relatively small) number of common dynamic factors, driven by common structural economic disturbances. The dynamics of the observed variables not due to the common factors are attributed to idiosyncratic (country-specific) shocks, uncorrelated with the common disturbances. This section describes the specification of the factor vector autoregressive model in more detail and provides some discussion of the adopted estimation methodology.

\subsection{Specification of the Factor Vector Autoregressive model}

The econometric model is composed of two sets of equations. The first refers to the "domestic" US economy (with variables collected in vector $\mathbf{X}_{t}$ ), while the second to the other $m-1$ "foreign", non-US countries $\left(\mathbf{Y}_{t}\right)$. The joint dynamics of $q$ macroeconomic variables for each of the $m$ countries of interest (in vector $\mathbf{Z}_{t}=\left[\mathbf{X}_{t} \mathbf{Y}_{t}\right]^{\prime}$ ) are modelled by means of the following reduced form dynamic factor model:

$$
\begin{aligned}
\mathbf{F}_{t} & =\boldsymbol{\Phi}(L) \mathbf{F}_{t-1}+\boldsymbol{\eta}_{t} \\
\mathbf{G}_{t} & =\boldsymbol{\Psi}(L) \mathbf{G}_{t-1}+\boldsymbol{\zeta}_{t} \\
\left(\mathbf{Z}_{t}-\boldsymbol{\mu}_{t}\right) & =\boldsymbol{\Lambda} \mathbf{F}_{t}+\boldsymbol{\Xi} \mathbf{G}_{t}+\mathbf{D}(L)\left(\mathbf{Z}_{t-1}-\boldsymbol{\mu}_{t-1}\right)+\mathbf{v}_{t}
\end{aligned}
$$

In (3) $\mathbf{Z}_{t} \sim I(0)$ is the $n \times 1$ stationary vector of variables of interest, with $n=m \times q$, and $\boldsymbol{\mu}_{t}=\left[\begin{array}{ll}\boldsymbol{\mu}_{t}^{X} & \boldsymbol{\mu}_{t}^{Y}\end{array}\right]^{\prime}$ is a $n \times 1$ vector of deterministic components, 
including an intercept term, and linear or non linear trends components. $\mathbf{F}_{t}$ is a $r \times 1$ vector of (observed or unobserved) common factors, generated by the stationary autoregressive process in (1) where $\boldsymbol{\Phi}(L)$ is a $r \times r$ finite order matrix lag polynomial, and $\boldsymbol{\eta}_{t}$ is a vector of shocks driving the $\mathbf{F}_{t}$ factors. $\mathbf{G}_{t}$ is a $s \times 1$ vector of stationary foreign factors, generated by the autoregressive process in (2) where $\boldsymbol{\Psi}(L)$ is a $s \times s$ finite order matrix lag polynomial, and $\boldsymbol{\zeta}_{t}$ is a vector of disturbances driving the $\mathbf{G}_{t}$ factors. The effects of both sets of factors on the US and non-US variables in $\mathbf{Z}_{t}$ are captured by the loading coefficients collected in the matrices $\boldsymbol{\Lambda}=\left[\begin{array}{ll}\boldsymbol{\Lambda}^{X} & \boldsymbol{\Lambda}^{Y}\end{array}\right]^{\prime}$ and $\boldsymbol{\Xi}=\left[\begin{array}{ll}\boldsymbol{\Xi}^{X} & \boldsymbol{\Xi}^{Y}\end{array}\right]^{\prime}$ (of dimension $n \times r$ and $n \times s$, respectively). Finally, $\mathbf{D}(L)$ is a $n \times n$ finite order matrix lag polynomial, partitioned as

$$
\mathbf{D}(L)=\left[\begin{array}{cc}
\mathbf{D}_{X X}(L) & \mathbf{0} \\
q \times q & q \times(m-1) q \\
\mathbf{D}_{Y X}(L) & \mathbf{D}_{Y Y}(L) \\
(m-1) q \times q & (m-1) q \times(m-1) q
\end{array}\right]
$$

with

$$
\mathbf{D}_{Y Y}(L)=\left[\begin{array}{cccc}
\underset{\mathbf{d}_{11}(L)}{q \times q} & \mathbf{0} & \ldots & \mathbf{0} \\
\mathbf{0} & \mathbf{d}_{22}(L) & \ldots & \mathbf{0} \\
\vdots & \ldots & \ddots & \vdots \\
\mathbf{0} & \mathbf{0} & \ldots & \mathbf{d}_{m-1, m-1}(L)
\end{array}\right]
$$

and $\mathbf{v}_{t}=\left[\begin{array}{ll}\mathbf{v}_{t}^{X} & \mathbf{v}_{t}^{Y}\end{array}\right]^{\prime}$ is the $n \times 1$ vector of reduced-form idiosyncratic (i.e. country-specific) disturbances. It is assumed that all polynomial matrices $\boldsymbol{\Phi}(L), \boldsymbol{\Psi}(L)$, and $\mathbf{D}(L)$ have all roots outside the unit circle. Moreover, $E\left[\eta_{j t} v_{i s}\right]=0, E\left[\eta_{j t} \zeta_{i s}\right]=0$ and $E\left[\zeta_{j t} v_{i s}\right]=0$ for all $i, j, t, s$.

The specification of the model in (1)-(5) embeds a set of important assumptions on the structure of linkages across countries: $(i)$ US idiosyncratic shocks $\left(\mathbf{v}_{t}^{X}\right)$ do not only affect the US economy (through $\mathbf{D}_{X X}(L)$ ), but also have spillovers on foreign countries (through $\mathbf{D}_{Y X}(L)$ ); $(i i)$ differently, foreign idiosyncratic disturbances $\left(\mathbf{v}_{t}^{Y}\right)$ do not affect US variables, while only own-country linkages are relevant for non-US economies $\left(\mathbf{D}_{Y Y}(L)\right.$ is block diagonal). The selected specification is then consistent with the view that the US play a leading role in the transmission of macroeconomic shocks; however, this does not prevent feedbacks from the rest of the world to the US economy, which are parsimoniously described by means of the foreign, non-US factors $\mathbf{G}_{t}$ which contribute to shape macroeconomic dynamics in all countries.

By substituting (1) and (2) into (3), the dynamic factor model can be 
written in standard vector autoregressive (VAR) form as

$$
\left(\begin{array}{c}
\mathbf{F}_{t} \\
\mathbf{G}_{t} \\
\left(\mathbf{Z}_{t}-\boldsymbol{\mu}_{t}\right)
\end{array}\right)=\left(\begin{array}{ccc}
\mathbf{\Phi}(L) & \mathbf{0} & \mathbf{0} \\
\mathbf{0} & \boldsymbol{\Psi}(L) & \mathbf{0} \\
\boldsymbol{\Lambda}(L) & \boldsymbol{\Xi} \boldsymbol{\Psi}(L) & \mathbf{D}(L)
\end{array}\right)\left(\begin{array}{c}
\mathbf{F}_{t-1} \\
\mathbf{G}_{t-1} \\
\left(\mathbf{Z}_{t-1}-\boldsymbol{\mu}_{t-1}\right)
\end{array}\right)+\left(\begin{array}{c}
\varepsilon_{t}^{F} \\
\varepsilon_{t}^{G} \\
\varepsilon_{t}^{Z}
\end{array}\right)
$$

where

$$
\left(\begin{array}{c}
\varepsilon_{t}^{F} \\
\varepsilon_{t}^{G} \\
\varepsilon_{t}^{Z}
\end{array}\right)=\left(\begin{array}{c}
\mathbf{I} \\
\mathbf{0} \\
\boldsymbol{\Lambda}
\end{array}\right) \boldsymbol{\eta}_{t}+\left(\begin{array}{c}
\mathbf{0} \\
\mathbf{I} \\
\mathbf{\Xi}
\end{array}\right) \boldsymbol{\zeta}_{t}+\left(\begin{array}{c}
\mathbf{0} \\
\mathbf{0} \\
\mathbf{v}_{t}
\end{array}\right)
$$

or, more compactly

$$
\mathbf{Z}_{t}^{*}=\mathbf{H}^{*}(L) \mathbf{Z}_{t-1}^{*}+\varepsilon_{t},
$$

with $\mathbf{Z}_{t}^{*}=\left[\begin{array}{lll}\mathbf{F}_{t} & \mathbf{G}_{t} & \mathbf{Z}_{t}-\boldsymbol{\mu}_{t}\end{array}\right]^{\prime}$, and variance-covariance matrix

$$
E\left(\varepsilon_{t} \varepsilon_{t}^{\prime}\right)=\Sigma_{\varepsilon}=\left(\begin{array}{ccc}
\boldsymbol{\Sigma}_{\eta} & \mathbf{0} & \boldsymbol{\Sigma}_{\eta} \Lambda^{\prime} \\
\mathbf{0} & \boldsymbol{\Sigma}_{\zeta} & \boldsymbol{\Sigma}_{\zeta} \boldsymbol{\Xi}^{\prime} \\
\Lambda \Sigma_{\eta} & \boldsymbol{\Xi} \Sigma_{\zeta} & \Lambda \Sigma_{\eta} \Lambda^{\prime}+\boldsymbol{\Xi} \Sigma_{\zeta} \Xi^{\prime}+\Sigma_{v}
\end{array}\right)
$$

where $E\left(\boldsymbol{\eta}_{t} \boldsymbol{\eta}_{t}^{\prime}\right)=\boldsymbol{\Sigma}_{\eta}, E\left(\mathbf{v}_{t} \mathbf{v}_{t}^{\prime}\right)=\boldsymbol{\Sigma}_{v}$ and $E\left(\boldsymbol{\zeta}_{t} \boldsymbol{\zeta}_{t}^{\prime}\right)=\boldsymbol{\Sigma}_{\zeta}$. Finally, the FVAR form in (7) can be inverted to obtain the following reduced-form vector moving average $(V M A)$ representation for the $\mathbf{Z}_{t}^{*}$ process:

$$
\mathbf{Z}_{t}^{*}=\mathbf{H}(L) \varepsilon_{t}
$$

where $\mathbf{H}(L)=\left(\mathbf{I}-\mathbf{H}^{*}(L) L\right)^{-1}$. The $V M A$ form describes the impulse responses of the variables in $\mathbf{Z}_{t}^{*}$ to the factor disturbances and idiosyncratic shocks in all countries.

The shocks in $\varepsilon_{t}$ have the nature of reduced-form innovations, and are linear combinations of the underlying structural disturbances driving the factors in $\mathbf{F}_{t}$ and $\mathbf{G}_{t}$ and the country-specific dynamics due to idiosyncratic shocks. In order to investigate the transmission within the US economy of several structural disturbances is then necessary to impose identification schemes in order to extract the relevant structural shocks from the reduced-form factor disturbances in $\boldsymbol{\eta}_{t}$ and $\boldsymbol{\zeta}_{t}$, and from the vector of US-specific disturbances $\mathbf{v}_{t}^{X}$. To this aim, we impose a set of exclusion restrictions on the contemporaneous responses of the factors and the US variables to the structural disturbances, implying a precise "ordering" for the elements in the $\mathbf{F}_{t}, \mathbf{G}_{t}$ and $\mathbf{X}_{t}$ vectors, based on plausible assumptions on the relative speed of adjustment to shocks. This identification strategy is described in detail in the Appendix.

Finally, in order to investigate the consequences of unanticipated changes in US macroeconomic dynamics on foreign countries (i.e. the spillovers from 
the US to other economies), we rely directly on the impulse response functions obtained from the reduced form F-VAR representation in (6), which is appropriate when the focus is on the impact of a change in a given forcing variable, say the US GDP growth rate, on the macroeconomic variables of all foreign countries independently of the underlying economic cause (i.e. a given structural shock). More precisely, the impact of a change in the variables of interest (the common factors in $\mathbf{F}_{t}, \mathbf{G}_{t}$ and the US series in $\mathbf{X}_{t}$,) on the non-US variables in $\mathbf{Y}_{t}$ is obtained from the relevant block of (6):

$$
\begin{aligned}
\mathbf{Y}_{t}-\boldsymbol{\mu}_{t}^{Y}= & \boldsymbol{\Lambda}^{Y} \boldsymbol{\Phi}(L) \mathbf{F}_{t-1}+\boldsymbol{\Xi}^{Y} \boldsymbol{\Psi}(L) \mathbf{G}_{t-1}+\mathbf{D}_{Y X}(L)\left(\mathbf{X}_{t-1}-\boldsymbol{\mu}_{t-1}^{X}\right) \\
& +\mathbf{D}_{Y Y}(L)\left(\mathbf{Y}_{t-1}-\boldsymbol{\mu}_{t-1}^{Y}\right)+\boldsymbol{\varepsilon}_{t}^{Y}
\end{aligned}
$$

by computing the dynamic multipliers, i.e.

$$
\mathbf{Y}_{t}-\boldsymbol{\mu}_{t}^{Y}=\mathbf{V}(L)\left(\begin{array}{c}
\mathbf{F}_{t-1} \\
\mathbf{G}_{t-1} \\
\mathbf{X}_{t-1}-\boldsymbol{\mu}_{t-1}^{X}
\end{array}\right)+\varepsilon_{t}^{Y}
$$

where $\mathbf{V}(L)=\left[\mathbf{I}-\mathbf{D}_{Y Y}(L) L\right]^{-1}\left(\boldsymbol{\Lambda}^{Y} \boldsymbol{\Phi}(L) \quad \boldsymbol{\Xi}^{Y} \boldsymbol{\Psi}(L) \quad \mathbf{D}_{Y X}(L)\right)$.

\subsection{Estimation}

As in Stock and Watson (2005), estimation is carried out by solving iteratively the following minimization problem

$$
\min _{\left\{\mathbf{F}_{j}, \mathbf{G}_{j}\right\}_{j=1}^{T}, \mathbf{\Xi}, \mathbf{\Lambda}, \mathbf{D}(L)} T^{-1} \sum_{t=1}^{T} \mathbf{v}_{t}^{\prime} \mathbf{v}_{t}
$$

where $\mathbf{v}_{t}=\left(\mathbf{Z}_{t}-\boldsymbol{\mu}_{t}\right)-\boldsymbol{\Lambda} \mathbf{F}_{t}-\boldsymbol{\Xi} \mathbf{G}_{t}-\mathbf{D}(L)\left(\mathbf{Z}_{t-1}-\boldsymbol{\mu}_{t-1}\right)$ from (3) and $T$ is the sample size. The methodology consists of two main steps.

First, as in the current application the common factors in $\mathbf{F}_{t}$ are directly observed, only a consistent preliminary estimate of the foreign factors in $\mathbf{G}_{t}$ is needed. This may be obtained by the application of principal components analysis (PCA) to the properly filtered non-US series in $\mathbf{Y}_{t}$. In particular, in order to ensure orthogonality with the $\mathbf{F}_{t}$ and $\mathbf{X}_{t}$ components, the filtered $\mathbf{Y}_{t}$ series $\left(\mathbf{Y}_{t}^{*}\right)$ are computed from the residuals of the regression of the actual $\mathbf{Y}_{t}$ series on its own lags (respecting the own country constraint in (5)), lagged $\mathbf{X}_{t}$ series and lagged $\mathbf{F}_{t}$ factors. Then, the $\mathbf{Y}_{t}^{*}$ vector is partitioned into categories of variables, and the common factors are estimated sequentially as the first principal component for each sub-set of series. Therefore, the $s$ static 
factors in $\mathbf{G}_{t}$ are separately estimated as the first principal components from the relevant sub-sets of variables, each including $m-1$ series. ${ }^{4}$ For instance, a "foreign GDP factor" is estimated as the first principal component from the set of the GDP growth series of the non-US countries under study.

Second, since each equation in the model does not contain the same set of regressors, OLS estimation is not as efficient as FIML. Yet, given the number of equations involved in the current application, the latter is infeasible. A gain in efficiency can however be achieved through iterated estimation as in Stock and Watson (2005), adopting the following procedure:

1. given the preliminary estimate for the $\mathbf{G}_{t}$ factor obtained as described above, we estimate the model in (3) by OLS to obtain an initial estimate of the $\mathbf{D}(L), \boldsymbol{\Xi}$ and $\boldsymbol{\Lambda}$ matrices;

2. conditional to the estimate of the $\mathbf{D}(L), \boldsymbol{\Xi}$ and $\boldsymbol{\Lambda}$ matrices, a new estimate of the $\mathbf{G}_{t}$ factors is obtained from PCA applied to the new filtered variables $\left(\mathbf{Y}_{t}-\hat{\boldsymbol{\mu}}_{t}^{Y}\right)-\hat{\boldsymbol{\Lambda}} \mathbf{F}_{t}-\hat{\mathbf{D}}(L)\left(\mathbf{Z}_{t-1}-\hat{\boldsymbol{\mu}}_{t-1}\right)$; conditional on these new estimated factors, updated estimates of $\boldsymbol{\Lambda}, \boldsymbol{\Xi}$ and $\mathbf{D}(L)$ can be obtained by OLS from (3).

This procedure is then iterated until convergence. Once the final estimate of $\mathbf{G}_{t}$ is available, the $\boldsymbol{\Psi}(L)$ matrix is obtained by applying OLS to (2); moreover the estimate of the $\boldsymbol{\Phi}(L)$ matrix is obtained by applying OLS to (1). Finally, the restricted VAR coefficients in (6) are obtained by employing the final estimates of $\boldsymbol{\Lambda}, \boldsymbol{\Xi}$ and $\mathbf{D}(L)$.

Although a formal proof is beyond the scope of this paper, it is conjectured that, based on the plug-in principle, the above estimation procedure should lead, at least, to consistent estimation of the parameters and quantities of interest. In fact, the procedure is based on the use of consistent and asymptotically normal estimators, as recent theoretical results also validate the use of PCA in the case of weakly dependent processes (Bai 2003) ${ }^{5}$, and the OLS estimator is consistent and asymptotically normal in the framework considered. This is also coherent with recent work by Pesaran and Chudik

\footnotetext{
${ }^{4}$ The sub-set strategy adopted here is preferable to the whole-set strategy (which uses all available variables) as it can make it easier to give an economic content to the factors, also avoiding contamination from series potentially unrelated to the phenomenon of interest. See Bagliano and Morana (2009) for additional details.

${ }^{5}$ Bai (2003) established consistency and asymptotic normality of PCA when both the unobserved factors and the idiosyncratic components show limited serial correlation, and the latter also display heteroschedasticity in both their time-series and cross-sectional dimensions.
} 
(2010) on high dimensional VARs with dominant unit. Moreover, albeit multi-step, the above procedure is iterated to improve efficiency. ${ }^{6}$

\section{The data}

We use seasonally adjusted quarterly macroeconomic time series data for the US and 30 advanced economies (Australia, Austria, Belgium, Canada, Czech Republic, Denmark, Finland, France, Germany, Greece, Hong Kong, Iceland, Ireland, Israel, Italy, Japan, Luxembourg, Netherlands, New Zealand, Norway, Portugal, Singapore, Slovakia, Slovenia, South Korea, Spain, Sweden, Switzerland, Taiwan, United Kingdom), 5 advanced emerging economies (Brazil, Hungary, Mexico, Poland, South Africa), and 14 secondary emerging economies (Argentina, Chile, China, Colombia, India, Indonesia, Malaysia, Morocco, Pakistan, Peru, Philippines, Russia, Thailand, Turkey), for a total of 50 countries. $^{7}$

For the US, our dataset includes real GDP, civilian employment, real private consumption, real private investment, fiscal deficit to GDP, current account deficit to GDP, CPI all items index, the three-month Treasury Bills real rate, the 10-year Federal government securities real rate, real house prices, the real effective exchange rate, and real share prices (S\&P500). Some additional financial variables have also been included, in order to capture financial distress (the TED spread, the AGENCY spread and the BAA-AAA corporate $\operatorname{spread}^{8}$ ) and liquidity conditions (the M2 to GDP ratio, and the total

\footnotetext{
${ }^{6}$ In the recent literature, several other approaches to global macroeconometric model estimation have been proposed. See Bagliano and Morana (2008) for a comparative discussion of the strengths and weaknesses of the procedure implemented here.

${ }^{7}$ US data are from FRED2 (Federal Reserve Bank of St. Louis); OECD countries data are from the OECD Main Economic Indicators, integrated with the IMF International Financial Statistics (bank loans series); data for the other countries are from the IMF International Financial Statistics; house price series for OECD countries are taken from a non official OECD database (see http://www.olis.oecd.org/olis/2006doc.nsf/linkto/ECOWKP282006293). The authors are grateful to P. Donati, S. Ejerskov, P. Benczur, M. Jensen for help in collecting some of the series.

${ }^{8}$ The TED spread is the spread between the 3 -month LIBOR rate (Euro-dollar deposit rate) and the yield on 3-month Treasury bills. Being the difference between an unsecured deposit rate and the risk-free rate, it can be taken as a measure of credit/liquidity risk. The Agency spread is the spread between agency (Freddie Mae, Fannie Mac) 30-year bonds and 30-year Treasury bonds, capturing the stress in the mortgage market. Finally, the BAA-AAA spread is the spread between corporate BAA and AAA bonds. In addition to being a measure of corporate default risk, it is also a measure of risk-taking, as a contraction of the spread implies an increase in the demand for riskier bonds relative to safer ones.
} 
loans and leases at commercial banks to GDP ratio). The time span of the US data is from 1980:1 to 2009:1, for a total of 117 observations.

Differently, a smaller set of variables has been considered for the other countries and collected in the $\mathbf{Y}_{t}$ vector. Due to data availability, non-US countries have been partitioned into two groups. The first group is composed of the 16 largest OECD economies (Australia, Canada, Japan, New Zealand, Denmark, Finland, France, Germany, Ireland, Italy, the Netherlands, Norway, Spain, Sweden, Switzerland, and the UK). For each of these countries 6 macroeconomic variables have been considered, including real GDP, the CPI-all items index, real bank loans to the private sector relative to GDP, real short-term interest rate (either a 3-month interbank rate or a 3-month Treasury Bills rate, depending on availability), and real house and stock prices. As for the US, the sample period runs from 1980:1 to 2009:1. The second group is composed of both advanced and emerging countries, for a total of 33 countries, including few European (OECD) economies (Austria, Belgium, Greece, Iceland, Luxembourg, Portugal), some Asian countries (Russia from Northern Asia, China, Hong Kong, Taiwan, and South Korea from Eastern Asia, Indonesia, Malaysia, Philippines, Singapore, and Thailand from Southeastern Asia, India and Pakistan from Southern Asia, and Israel and Turkey from Western Asia), some Latin American countries (Argentina, Brazil, Chile, Colombia, Mexico, and Peru), some emerging European countries (the Czech Republic, Hungary, Poland, Slovakia, and Slovenia); and one Northern (Morocco) and one Southern Africa countries (South Africa). Differently from the former group, only 5 macroeconomic variables have been considered for these economies, omitting the house price series, and a shorter sample period, 1995:1 through 2009:1, is employed. Despite the relatively small sample (56 observations) our parsimonious specification and (simulation-based) estimation strategy should grant reliability to the results.

The vector of (observed) common factors $\mathbf{F}_{t}$, affecting both the US and non-US economies, includes crude oil price and primary commodities (excluding energy) price shocks, constructed following the procedure set out by Hamilton (1996). ${ }^{9}$ Working with shock variables rather than with actual oil and commodity price series yields some advantages. First, it allows to account for the nonlinearity coming from the asymmetric impact of

\footnotetext{
${ }^{9}$ In Hamilton (1996), the oil shock is measured by the maximum of (a) zero and (b) the difference between the log-level of the series for the current quarter and the maximum value of the log value of the series in the previous four quarters. Hence, in order to be considered, an oil price increase needs to be large enough to off-set any decrease occurred over the previous year. This procedure is applied here both to oil prices and to a (exenergy) primary commodities price index.
} 
oil/commodity price shocks, with only negative shocks, i.e. price increases, affecting real activity. Second, it allows to account for the potential endogeneity problems of oil and commodity prices, which may be relevant for the largest economies in the sample, i.e. the US, China and the Euro Area. By graphical inspection, it can be concluded that the computed shock variables are consistent with two major episodes, which may be either associated with pure supply phenomena (the Iraq's invasion of Kuwait in August 1990), or with speculative or non-US demand (emerging Asia) developments. Hence, modelling oil and commodity shocks as exogenous may be appropriate given the sample considered and the scope of the paper.

\subsection{Persistence properties}

The persistence properties of the data have been assessed by means of the KPSS stationarity test, in the standard form (Kwiatkowski et al., 1992), as well as by allowing for a non linear trend under the null (Becker et al., 2006), in order to account for structural change. In the latter test the deterministic component $\mu_{t}$ is modelled by means of the Gallant (1984) flexible functional form, whereby $\mu_{t}=\mu_{0}+\mu_{1} t+\mu_{2} \sin (2 \pi t / T)+\mu_{3} \cos (2 \pi t / T)$, capturing not only various forms of non linear smooth deterministic trends, but also being able to account for the presence of sharp breaks.

The tests have been carried out directly on the series used in the empirical analysis, i.e. growth rates for employment (denoted by $e$ ) and real GDP $(g)$ and its components, i.e. private consumption $(c)$ and investment $(i)$; the rate of CPI inflation $(\pi)$; rates of growth for real house prices $(h)$, the real effective exchange rate $(e r)$, real stock prices $(f)$, as well as for excess real credit growth $(l o)$ (i.e. the spread between real liquidity growth and real GDP growth); the levels of the long-term and short-term real interest rates ( $l$ and $s$, respectively), the US current account to GDP ratio (cad), the US public deficit to GDP ratio $(p d)$, and the rate of change of two variables (discussed below) capturing financial fragility $(f r)$ and excess liquidity $(e x l)$ in the US.

The KPSS test is supportive of the selected stationary specification, pointing to $\mathrm{I}(0)$ stationarity for all series. Yet, particularly for inflation rates, and for most of the countries, stationarity seems to occur around the non-linear deterministic specification employed, favoring the inclusion of the latter in the model. ${ }^{10}$ These findings are consistent with previous results of Bagliano and Morana (2009) and Beltratti and Morana (2010), though for a smaller

\footnotetext{
${ }^{10}$ Detailed results are not included for reasons of space, but are available from the authors upon request.
} 
set of countries and a shorter sample, and may be rationalized in terms of successful long-term monetary policy management. Thus, on the basis of the above results, the stationary representation of the F-VAR model has been augmented by including the Gallant (1984) specification for the deterministic component.

\section{Model specification and estimation}

To reach the final specification for the vector of US variables, $\mathbf{X}_{t}, \mathrm{PCA}$ has been applied to sub-sets of the US series in order to determine whether different variables provide similar information that could be suitably summarized by common components, whereby avoiding potential multicollinearity problems. In particular, a common component, explaining about $80 \%$ of variability for each series, can be extracted from the BAA-AAA, AGENCY and TED spreads. Hence, only the first principal component extracted from those series has been retained, and interpreted as a financial fragility index $(f r)$ for the US economy. Figure 1(a) portrays the behavior of the three spreads and the constructed index over the estimation sample, showing two major peaks at the beginning of the 1980s and in 2008. Similarly, as a single component explains about $80 \%$ of the variability of excess M2 and bank loans (relative to GDP) growth, only their first principal component, interpreted as an excess liquidity index $(\mathrm{exl})$, has been included in the vector of US variables rather than the actual series. ${ }^{11}$ This index, displayed in Figure 1(b), captures the gradual build-up of liquidity that started around 1995 and accelerated over the period 2006-2008. Although other factors could likely be extracted from the set of US variables, i.e. a common real interest rate component from the short- and long-term real interest rates and a real activity factor from output, consumption and investment, in order to fully gauge the interactions among US variables, consistent with the aim of this study, the actual series have been employed.

Therefore, the US vector $\mathbf{X}_{t}$ includes 14 variables, in the following order: employment growth, real GDP growth, the federal deficit/GDP ratio, real private consumption growth, real private investment growth, the current account/GDP ratio, the CPI inflation rate, the excess liquidity index, the real three-month Treasury bills rate, the real ten-year Government Bonds rate, real house price returns, real effective exchange rate returns, real stock price returns, and the financial fragility index. The rationale for the chosen ordering is based on the variables' speed of adjustment to shocks, with a distinction

\footnotetext{
${ }^{11}$ Detailed results are available upon request from the authors.
} 
between relatively slow-moving variables (mainly related to real activity, ordered first) and fast-moving variables (notably financial quantities, ordered last).

Differently, a smaller set of variables is included in the $\mathbf{Y}_{t}$ vector for the non-US countries, namely real GDP growth, CPI inflation, real excess credit growth, the real short-term rate, real house price returns (when available), and real stock price returns, in that order. For parsimony reasons, only five lagged US variables (real GDP growth, the excess liquidity index, real house price returns, real stock price returns and the economic/financial fragility index) are included in the equations for the non-US series.

Concerning the non-US factors, a single common component has been included in the $\mathbf{G}_{t}$ vector, extracted from the GDP growth series of the 37 countries for which data are available since 1980:1, ${ }^{12}$ and capturing common movements in the (non-US) level of world economic activity. This component accounts for about $20 \%$ of total variance, with sizable differences in the proportion of GDP growth variance explained for each country, ranging from about $55 \%$ for Denmark to less than $1 \%$ for Indonesia. ${ }^{13}$ Figure 1(c) portrays the (standardized) non-US common GDP growth factor and the US GDP growth rate over the sample, and shows a sizable positive correlation (0.43) between the two series. Consistent with the procedure detailed in the methodological section, this component has been employed in the implementation of the first step of the iterative estimation procedure.

Finally, as already mentioned in the preceding section, Hamilton's (1996) shocks to oil price and (non-oil) primary commodities index have been included in the $\mathbf{F}_{t}$ vector.

\subsection{The F-VAR model: estimation}

On the basis of the BIC information criterion, the optimal lag length of the F-VAR system is set equal to one. Then, consistently with the Granger and Jeon (2004) thick modelling approach, up to three lags are considered in estimation, and median estimates for the parameters of interest are obtained through simulation (with 1000 replications).

The whole estimated system counts 278 equations. In particular, the 14

\footnotetext{
${ }^{12}$ This list includes the largest 18 OECD countries (Austria, Belgium, Denmark, Finland, France, Germany, Ireland, Italy, the Netherlands, Norway, Spain, Sweden, Switzerland, the UK, Australia, Canada, Japan and New Zealand), and a selection of the Latin American countries (Argentina, Brazil, Chile, Colombia, Mexico, Peru), Asian countries (China, Hong Kong, Korea, Taiwan, Indonesia, Malaysia, Philippines, Singapore, Thailand, India, Pakistan, Turkey) and African countries (Morocco, South Africa).

${ }^{13}$ Detailed results are available upon request from the authors.
} 
equations corresponding to the US block $\mathbf{X}_{t}$ contain a minimum (maximum) of 21 (65) parameters, of which 14 (52) are for the lagged US series, 3 (9) for the lagged $\mathbf{F}_{t}$ and $\mathbf{G}_{t}$ series, and 4 are for the deterministic component (including a constant, a linear trend and two trigonometric components, as described in data section). The vector $\mathbf{X}_{t}$ collects the 14 US endogenous macroeconomic variables (namely $e, g, p d, c, i, c a d, \pi, e x l, s, l, h, e r, f$ and $f r$, in this order).

Assuming an own-variable block diagonal structure for the corresponding elements of the $\mathbf{D}(L)$ matrix for the foreign countries, i.e. a diagonal $\mathbf{D}_{Y Y}(L)$ as in (5), the block of equations for the 16 largest OECD countries counts a total of 96 equations, each containing a minimum (maximum) of 13 (31) parameters, of which 1 (3) for the lagged own variable, 5 (15) are for the lagged US series, 3 (9) for the lagged $\mathbf{F}_{t}$ and $\mathbf{G}_{t}$ series, and 4 for the deterministic component. For each of the 16 above countries the vector $\mathbf{Y}_{t}$ collects 6 endogenous macroeconomic variables (namely $g, \pi, l o, s, h$, and $f$, in this order). The block of equations corresponding to the remaining 33 countries, counts a total of 165 equations, with similar specification. For each of the latter 33 countries the vector $\mathbf{Y}_{t}$ collects 5 endogenous variables (namely $g, \pi, l o, s$, and $f$, in this order). Finally, the last 3 equations describe the dynamics of the common factors (oil and commodities price shocks) and the non-US common GDP growth factor.

\section{Crisis dynamics in the US}

In this section, the estimated F-VAR model is used to explore the economic mechanisms that transmit various shocks hitting the US economy to a large set of domestic variables (collected in $\mathbf{X}_{t}$ ), in order to gain valuable insights on the complex interactions of economic and financial factors determining the dynamics of the current "Great Recession" episode. In particular, the impulse response functions obtained from the econometric model are analyzed to assess the coherence of the mechanics uncovered with the boom-bust credit cycle hypothesis. Operationally, the identification of the structural shocks has been achieved by means of a Choleski procedure (described in the Appendix) based on the variables' speed of adjustment to shocks, with a distinction between relatively slow-moving variables (mainly related to real activity, ordered first) and fast-moving variables (notably financial quantities, ordered last).

Concerning the slow-moving variables, the economic rationale behind the assumed recursive structure (going from employment to GDP growth, the public deficit to GDP ratio, consumption and investment growth, the cur- 
rent account to GDP ratio, and inflation), lies on the assumption that, over the business cycle, real activity is contemporaneously determined by employment (through a short-run production function), with the latter adjusting to the phase of the cycle only with (one-quarter) delay. Moreover, output contemporaneously determines private consumption (consumption function), investment (investment function) and net import, while the fiscal stance is adjusted according to output dynamics; private consumption and investment contemporaneously adjust to changes in the fiscal stance (either anticipating future output growth or due to Barro-Ricardo and/or crowding out effects), and net import is contemporaneously determined by the state of domestic demand; aggregate demand then feedbacks, with a (one-quarter) delay, to aggregate supply, and prices adjust according to aggregate demand and supply interactions.

On the other hand, concerning the fast-moving variables, the assumed ordering (going from excess liquidity to real short- and long-run interest rates, real house prices, the real exchange rate, real stock prices, and the financial fragility index) implies that liquidity conditions contemporaneously determine interest rates and asset prices, while liquidity may respond to asset prices developments only with a (one-quarter) delay. This is consistent with asset prices rapidly adjusting to the stance of monetary policy, with the Fed at most implementing a leaning-against-the-wind strategy, relatively to asset price dynamics; hence, the real short-term rate is contemporaneously determined by liquidity conditions, while the real long-term rate is contemporaneously determined by the real short-term rate. Real house prices and the real effective exchange rate are contemporaneously determined by liquidity conditions and interest rates, while real stock prices contemporaneously react to any change in the economy. Finally, the financial fragility index embeds all contemporaneous information on the state of the business cycle. Note also that the slow- to fast-moving ordering implies that monetary policy, the key determinant of liquidity and interest rates in the economy, is set according to the state of the business cycle.

Median forecast error variance decomposition and cumulated impulse responses to unitary shocks, with $90 \%$ significance bands, have been computed up to a horizon of three years, to show the dynamic reaction of the level of investigated variables. ${ }^{14}$ We start with a presentation of the result delivered by the forecast error variance decomposition analysis. As the Choleski approach may lead to policy conclusions which are not robust to the ordering of the variables, due to the contemporaneous correlation of their reduced form

\footnotetext{
${ }^{14}$ Only selected results have been reported for reasons of space. A full set of results is available upon request from the authors.
} 
disturbances, we discuss some robustness issues at the end of the section.

\subsection{Forecast error variance decomposition}

According to the results of the forecast error variance decomposition reported in Table 1, the overall picture is fairly consistent with standard macroeconomic theory. In particular, demand side shocks are more relevant for real activity than supply side (productivity) disturbances in the short-term and the latter gain importance over a medium-term horizon, private consumption shows quicker adjustments than investment, and financial shocks are more relevant for real activity fluctuations in the medium-term than in the short-term. Moreover, fluctuations in financial variables may be determined by both fundamentals (ultimately driven by consumption and productivity shocks) and purely speculative factors, with stock prices showing a larger speculative component than bond and house prices. Close interrelationships among financial assets are also detected, with the short-term interest rate being relevant for financial fragility and house price fluctuations, as well as excess liquidity dynamics. Hence, the overall picture appears to be consistent with a boom-bust credit cycle scenario, where financial factors are the triggering mechanism of the downturn in real activity and worsened economic conditions feedback to asset prices, starting a cumulative process. A selection of the most relevant results is presented in detail below.

\subsubsection{Real side fluctuations in the US}

Fluctuations in US real activity are mostly determined by real side shocks in the short-term, while financial factors may have some role in the mediumterm. In fact, in the short-term, real output responds only to the own (aggregate demand) (68\%), employment (23\%) and inflation (aggregate supply/productivity) (5\%) shocks; rather, in the medium-term the aggregate demand (44\%) and employment (11\%) disturbances loose somewhat importance, while the aggregate supply $(12 \%)$ and the short-term real interest rate shock (18\%) become more relevant. ${ }^{15}$ A coherent pattern can be detected for consumption and investment as well, with the employment and aggregate demand shocks having sizable effects at all forecasting horizons for both variables (13\% to $30 \%$ ), while the house price ( $7 \%$ to $8 \%$ ), the current account deficit (13\%), the financial fragility index (on consumption, $7 \%$ ) and the real short-term rate (on investment, 21\%) disturbances play a larger role in the

\footnotetext{
${ }^{15}$ The economic interpretation of the shocks is discussed in the subsequant section on impulse response analysis.
} 
medium-term. Finally, employment is strongly idiosyncratic, with the aggregate supply, aggregate demand and real short-term rate shocks contributing somewhat to fluctuations only at the three-year horizon $(8 \%, 13 \%$ and $17 \%$, respectively).

\subsubsection{Financial fluctuations in the US}

Concerning asset price volatility, a relevant role is played by consumption and productivity shocks, at all horizons. For instance, the contribution of the consumption shock to fluctuations of real short- and long-term rates is always sizable ( $15 \%$ to $21 \%$ ), while the productivity shock is actually dominant (40\% to $54 \%$ ); similarly for house prices (12\% to $29 \%$ ) and economic/financial fragility conditions (productivity, $14 \%$ to $22 \%$; consumption, $7 \%$ in the medium-term); for stock prices, as well as excess liquidity, somewhat less (productivity, $5 \%$ to $9 \%$; consumption, $4 \%$ to $11 \%$ ); for the latter variable also the aggregate demand shock plays an important role (14\% to $38 \%)$.

Yet, other disturbances also matter, albeit to a lower extent: the public deficit shock is relevant for interest rates (4\% to $7 \%$ ), stock prices $(6 \%$ in the very short-term), and economic and financial fragility conditions (4\% in the medium-term); the current account deficit shock matters for stock prices ( $11 \%$ to $20 \%$ ) and excess liquidity (20\% in the medium-term); employment disturbances are relevant for the short-term rate $(7 \%)$, as well as for economic/financial fragility in the medium-term (7\%); finally, the shortterm interest rate is important for financial fragility conditions ( $9 \%$ to 17\%), house prices ( $11 \%$ in the medium-term), and the long-term rate (mediumterm, $5 \%$ ).

\subsubsection{Fluctuations in US domestic and foreign debt}

Both the fiscal deficit/GDP ratio and the current account deficit/GDP ratio are strongly idiosyncratic at the two-quarter horizon $(80 \%$ and $88 \%$, respectively), but somewhat less in the medium-term, as employment, house prices, productivity and interest rate shocks play some role. For instance, figures for medium-term fluctuations in the fiscal deficit/GDP ratio are $5 \%$ to $7 \%$ for employment, house price and real short-term rate shocks; similar figures are found for the current account deficit/GDP ratio, i.e. about $5 \%$ for the aggregate demand, house price and productivity shocks, while short- and long-term rates disturbances have a more sizable effect (13\% to 16\%). Hence, our results point to a much weaker role of stock and house prices in determining US current account deficit fluctuations than found by Fratzscher et 
al. (2009).

\subsection{Impulse response analysis}

Concerning the mechanics of the crisis, the results of the impulse response analysis allow to draw relatively clear-cut conclusions. Table 2 reports the median cumulated responses of the US variables to unitary shocks over a two-quarter, one-year and three year-horizons; significant figures at the $10 \%$ level, are shown in bold.

\subsubsection{Financial linkages}

According to a boom-bust credit cycle interpretation of the crisis, asset prices misalignments in the housing and stock markets are initially fuelled by the availability of excess liquidity and low interest rates. As shown in Table 2, no significant connection can be detected between excess liquidity shock and house and stock prices, though the point medium-term impact on stock prices is sizable $(0.15 \%)$. Yet, following a positive shock to excess liquidity, the short- and long-term rates decrease (by 14 basis points), with a temporary contraction in the real short-term interest rate then leading to a significant increase in house $(0.6 \%$ in the medium-term) and stock prices $(0.9 \%$ in the very short-term).

Different economic mechanisms can explain the correlations between asset prices, interest rates and liquidity, providing a causal interpretation of the observed linkages. Portfolio rebalancing would predict a positive relationship between excess liquidity and asset prices, as the increased liquidity would be allocated to the various assets, increasing their demand and price; moreover, from the present value model, a reduction in the interest rate leads to lower discounting of the flow of expected future dividends (rents), increasing stock (house) prices; finally, a contraction in the mortgage rate can ease liquidity constraints, boosting housing demand and prices (Alm and Follain, 1984). Other linkages may also operate, as higher asset prices may boost the value of firms' collateral, increasing their borrowing ability, and at the same time improving the balance sheets of financial institutions and increasing leverage. Financial accelerator mechanisms may also amplify the above effects, fuelling an asset price-balance-sheet-credit spiral. The significance of feedback effects from stock prices to liquidity becomes apparent if, rather than focusing on excess liquidity, just liquidity $(l o)$ is considered. As exl $=l o-g$, the response of liquidity to a stock prices increase can be obtained as $l o=e x l+g$, yielding $0.05 \%, 0.36 \%$ and $0.83 \%$ at the $2-, 4$ - and 12 -quarter horizons, respectively. 
Concerning the generation of excess liquidity, a potential role can finally be ascribed to the current account deficit, consistent with the view that huge US trade deficits contributed to the boom phase of the credit cycle, fostering growth of global liquidity and further debt accumulation. As reported in Table 2, an increase in the current account deficit/GDP ratio leads in fact to a sizable increase in excess liquidity $(0.4 \%)$ and depreciation of the real exchange rate $(-0.84 \%)$, with a negative medium-term impact on real activity (consumption, $-0.3 \%$; investment, $-0.8 \%$ ) and stock prices $(-2.7 \%) .{ }^{16}$

The setting-in of the bust phase of the current crisis might have followed expected, but not materialized, house price appreciation, which would have lead the predatory lending mechanism to break down and to a generalized decline in asset prices and tight credit conditions, as financial institutions were forced into deleveraging and recapitalization. While our empirical evidence on the asset prices-balance-sheet-credit spiral is weak, the positive and significant correlation between house and stock prices may indeed be useful to describe the effects of the deleveraging process, with a negative house price shock leading to a contraction in stock prices $(-1.3 \%)$ in the short-term (flexible adjustment), and a negative stock price disturbance leading to a contraction in house prices $(-0.3 \%)$ in the medium-term (sluggish adjustment). Yet, the correlation is also consistent with a portfolio model where prices depend on net inflows. Then, a change in wealth determined by a contraction in house (stock) prices would lead agents to rebalance their portfolios by selling stocks (housing) as well (see Beltratti and Morana 2010 for similar findings).

\subsubsection{Real effects of the crisis}

Concerning the real effects of the crisis, different theoretical relationships can be envisaged between asset prices and credit conditions and real activity.

First, tight credit conditions may constrain consumption and investment expenditure (Gauger and Snyder, 2003; Leamer, 2007; Greenlaw et al., 2008; Dell'Ariccia et al., 2008; Bayoumi and Mellander, 2008; Goodhart and Hoffman, 2008; Schularick and Taylor, 2009). Our empirical evidence is not clear-cut on this issue, pointing to a positive correlation between excess liquidity and employment $(0.02 \%)$, as well as to a negative correlation between excess liquidity and investment $(-0.11 \%)$ in the very short-term.

Second, falling asset prices may affect real activity also through wealth

\footnotetext{
${ }^{16}$ According to Jagannathan et al. (2009), behind US trade dynamics there would however be inadequate financial markets, preventing higher levels of domestic consumption and investment in emerging economies, as well as currency controls, motivated by exportled growth objectives, particularly in China.
} 
effects on consumption and Tobin's "q" effects on investment. According to the life-cycle model, a permanent increase in housing wealth leads in fact to an increase in spending and borrowing by homeowners, as they try to smooth consumption over the life cycle. The increase in property value actually enables them to borrow more as it increases the value of collateral. Additional effects can be expected through Tobin's "q" effects, as an increase in house prices determines an increase in property value over construction costs, stimulating residential investment. Our empirical evidence is fairly consistent with the above channels. A negative house price shock does indeed lead to a significant contraction in consumption (up to $-0.2 \%$ ) and investment (up to $-0.6 \%$ ), as well as in output and employment in the short-term. Similar evidence is found for a negative stock price disturbance, though with a weaker impact $(-02 . \%$ and $-0.1 \%$ for consumption and investment, respectively). Overall, the findings are consistent with previous evidence in the literature, pointing to an inelastic impact of asset prices on real activity, and stronger for house prices than for stock prices (Beltratti and Morana, 2010; Bagliano and Morana, 2008; Case et al., 2005; Chirinko et al., 2004; Carrol et al., 2006).

Finally, while the credit boom phase occurred in an environment of low inflation, a deflation risk was actually perceived after the bust. From Table 2 a linkage between asset price busts and inflation can be established, with a negative house price shock leading to a significant contraction in consumer prices in the short-term $(-0.12 \%)$.

\subsubsection{Feedbacks from the real to the financial side}

Second-round effects from the downturn in real activity on asset prices can also be expected. The empirical evidence is not fully clear-cut, as a negative aggregate demand shock ${ }^{17}$ leads to a (not significant) contraction in house prices in the medium-term and in stock prices at all horizons (significant only in the short-term). On the other hand, a stronger impact is attributable to the aggregate supply (productivity) shock ${ }^{18}$, which is significant at

\footnotetext{
${ }^{17}$ The output shock, due to the positive short-term median correlation with inflation and interest rate, is interpreted in terms of aggregate demand shock.

${ }^{18}$ The inflation shock, in our framework, as in Bagliano and Morana (2009), may bear the interpretation of productivity shock. The argument follows from the fact that the structural inflation shock is estimated from dynamics around the non linear deterministic trend, which can be related to the disinflationary policy carried out by the Fed over the 1980s, and the successful inflation control thereafter, i.e. to long-term monetary policy management. The proposed interpretation is consistent with the results in Table 2, showing that a negative productivity shock (positive inflation shock) would lead to an increase in the price level and a contraction in output, as well as with Gordon (2005),
} 
any horizon, with a $1 \%$ medium-term output contraction, following a negative productivity shock, being associated with a $2.5 \%$ reduction in house prices and with a $8 \%$ contraction in stock prices over the same horizon. A present value model, relating future developments in dividends and rents to output dynamics, could account for the observed features, as a negative productivity shock (positive inflation shock), by decreasing dividends and rents, and increasing the discount factor (due to the inflation component in nominal interest rates), would lead to a contraction in asset prices. ${ }^{19}$

\subsubsection{The role of external demand}

From the US the crisis has quickly spread to the other economies. Hence, second-round effects may be expected through external demand. As shown in Table 2, a foreign output contraction has a negative and significant impact on US real activity ( $0.10 \%$ reduction in output in the short-term) and employment (up to $0.07 \%$ ), leading to a short-term increase in the fiscal deficit $(0.04 \%)$ and excess liquidity $(0.13 \%)$, and to an improvement in the current account $(-0.07 \%)$. Moreover, a short-term contraction in consumer prices ($0.05 \%)$, as well as in stock prices $(-4.5 \%)$, is observed. Hence, second-round effects should not be neglected when assessing the real costs of the financial crisis for the US economy. ${ }^{20}$

pointing to an important role of productivity growth for US inflation dynamics.

${ }^{19}$ The identification of global (US) aggregate demand and supply shocks, as well as of a monetary policy shock related to the short-term rate management by the Fed, is consistent with the results of Dees et al. (2010), also implementing a multi-country macroeconometric moodel. Yet, in terms of the size of the shocks our results are not fully comparable with those of Dees et al. (2010), as rather than using cyclical deviations from trends, actual rates of growth and returns are employed in the current study. It is however worthwhile mentioning that, considering the 1-year horizon, a 20 b.p. increase in the US real shortterm rate would lead to a contraction in US real output of similar size, i.e. about $-0.4 \%$ (relative to trend) in Dees et al. (2010) and $-0.3 \%$ with our approach. Moreover, a positive aggregate demand shock, leading to a $3 \%$ increase in US GDP (relative to trend) would lead the nominal US short-term rate to increase of about 80 b.p. (relatively to trend) in Dees et al. (2010) and of about 60 b.p. with our approach. Figures concerning the aggregate supply shock are, on the other hand, somewhat different, with a negative aggregate supply shock, leading to a $1 \%$ increase in the US price level, being associated with a $-2 . \%$ contraction in real output (relative to trend) in Dees et al. (2010), but only with a $-0.5 \%$ decrease with our approach.

${ }^{20}$ The empircal relevance of feedback effects from the world (non-US driven) business cycle to the US business cycle is not inconsistent with the fact that the US is a major driver in world output fluctuations. In fact, albeit being a net importer, the US is still one of the world top exporters in machinery and equipment, industrial supplies, non-auto consumer goods, motor vehicles, aircraft, food, feed and beverages. Its main export partners are Canada, the European Union, Mexico, China and Japan. 


\subsubsection{The effects of economic policy}

As the crisis was unfolding, an expansionary fiscal/monetary policy mix was implemented, ${ }^{21}$ in order to contrast its effects on real activity and ensuring the survival of the financial system. From Table 2 it can be noted that a negative aggregate demand shock would in fact lead to a significant short-term increase in excess liquidity $(0.4 \%)$, as well as to contraction in the short-term real interest rate (by 10 basis points) in the short-term. Similar dynamics are also observed in the aftermath of a negative employment shock, which also triggers an expansionary fiscal policy in the very short-term. Similarly, the implementation of an expansionary fiscal policy would also be triggered by a positive shock to the financial fragility index (i.e. an increase in liquidity/credit risk, corporate risk/risk appetite, stress in the mortgage market), to which a positive response of real activity (consumption, $0.2 \%$ ) may be associated, as well as an improvement in stock (0.3\%) and house prices $(0.2 \%)$. The effectiveness of the expansionary fiscal policy is also supported by the significant medium-term expansion of output (0.2\%) and employment $(0.1 \%)$, following a positive fiscal deficit shock. The presence of idle resources in the economy, i.e. unemployed labour and underutilized capital, liquidity constraints and low interest rates, which can make crowding out effects on private spending negligible, may explain the findings. A significant expansionary impact on output $(0.3 \%)$ and real activity, as well as on house $(0.6 \%)$ and stock prices $(0.9 \%$ in the short-term), is also triggered by a short-term rate cut, pointing to the effectiveness of an expansionary monetary policy implemented through the standard interest rate channel. Overall, our findings are consistent with Almunia et al. (2009), pointing to the effectiveness of fiscal and monetary policies during the Great Depression, where macroeconomic conditions were close to those currently prevailing in the major world economies, as well as with Buiter (2009), pointing to the effectiveness of interest rate policies, while quantitative and credit easing strategies would have failed at avoiding credit crunch effects.

\footnotetext{
${ }^{21}$ Surely peculiar to the current crisis is the rescue plan launched by the US government, i.e. the Troubled Asset Relief Programme (TARP), which has taken various forms of liquidity injection and debt relief programmes, aiming at defreezing the interbank and repo markets, and easing the banking sector from the burden of the unperforming loans, as well as to facilitate its recapitalization, even through its partial nationalization (capital injection through purchase of equity by the government). At the same time the Fed has implemented both conventional interest rate policies, as well as less conventional quantitative and credit easing strategies in order to ensure the provision of liquidity in the banking sector and in the overall economy. See Reis (2009) for an accurate account of the policies implemented by the Fed during the crisis.
} 


\subsubsection{The contribution of adverse supply shocks}

The response of the macroeconomy to oil and commodity price shocks is consistent with expectations. In fact, an oil price hike has a "stagflationary" effect, leading to a contraction in real activity (-0.8\%) and employment ($0.5 \%)$ in the medium-term, and to an increase in the general price level (1.1\%). Accommodation of the shock is observed, with sizable interest rates cuts (by 286 basis points), in the face of falling asset prices (-3.6\% and $-18 \%$ for house and stock prices, respectively). The current account deficit also worsens in the short-term, consistently with the increased oil price. Similar dynamics is observed also for commodity prices.

\subsubsection{Determinants of economic and financial fragility}

Finally, concerning the financial fragility index, some interesting conclusions can be drawn from its response to various structural shocks. First, negative productivity and negative aggregate demand disturbances lead to a significant increase in fragility in the short-term. Sizable and significant positive short-term impacts on fragility can also be associated with a short-term rate increase and oil and commodity price hikes. Finally, a positive excess liquidity shock would also lead to an increase in the financial fragility index in the short-term. Hence, the latter variable may be retained as a summary measure of incoming financial stress, complementary to the observation of house and stock price dynamics. Yet, it is worthwhile noting that the financial fragility index used in this paper shares some of the properties of the leading indicator for an incoming bust phase proposed by Borio (2008), which exploits the joint occurrence of rapid credit growth and higher risk taking, but not increasing asset prices.

\subsection{Robustness issues}

The chosen ordering of the US variables is based on two main assumptions: (i) supply-side disturbances have a contemporaneous effect on aggregate demand components, while demand feedbacks to supply with a (one-quarter) delay; (ii) liquidity conditions determine contemporaneously the short-term real interest rate, while the latter feedbacks to liquidity conditions only with a (one-quarter) delay. In order to assess the robustness of the policy conclusions drawn in this Section to the above assumptions, the analysis has been repeated for a different ordering the variables, inverting the contemporaneous role of supply and demand, and liquidity and the short-term rate. In particular, for the slow-moving variables the following alternative ordering 
is considered: consumption, investment, public deficit to GDP ratio, current account deficit to GDP ratio, output, employment and inflation; on the other hand, for the fast-moving variables the alternative ordering is: real short-term interest rate, excess liquidity, real long-term interest rate, real house prices, real effective exchange rate, real stock prices and the financial fragility index.

As shown in Table 1A in the Appendix, the results of the impulse response analysis are remarkably robust to the ordering reversal considered, as no major changes concerning median responses can in general be noted. There are however two interesting differences between the results reported in Table 2 and in Table 1A, which is worthwhile mentioning. Firstly, the median response of real activity to an employment shock, and of domestic demand to an output/aggregate demand shock, although of the same sign, is more muted than what found for the original ordering; secondly, the output/aggregate demand shock would seem to be deflationary and neutral on stock prices. Concerning the latter feature, our results show that, for the modified ordering, it is the consumption shock (not reported) which should probably bear the interpretation of aggregated demand shock, positively affecting output, the price level, interest rates and stock prices.

\section{Spillovers effects outside the US}

Rather than reporting results on a country by country basis, figures in Table 3 refer to descriptive statistics of the cross-country distribution of the dynamic multipliers, at the 2-quarter (short-term) and 12-quarter (mediumterm) horizons, for selected foreign variables (output, excess credit, house prices and stock prices), of OECD (+ Israel) and non-OECD economies, following US unitary percentage changes in output, excess liquidity, house and stock prices, and financial fragility. ${ }^{22}$

\footnotetext{
${ }^{22}$ In this study the OECD group includes 32 currently member countries (Australia, Austria, Belgium, Canada, Czech Republic, Denmark, Finland, France, Germany, Greece, Hungary, Iceland, Ireland, Italy, Japan, Korea, Luxembourg, Mexico, Netherlands, New Zealand, Norway, Poland, Portugal, Slovak, Slovenia, Republic, Spain, Sweden, Switzerland, Turkey, United Kingdom, United States) and Israel, which is about to join. The non-OECD group is then composed of the remaining 17 countries (Argentina, Brazil, Colombia, Mexico, Peru, China, Hong Kong, Taiwan, Indonesia, Malaysia, Philippines, Singapore, Thailand, India, Pakistan, Morocco and South Africa).
} 


\subsection{Responses to US output dynamics}

As shown in Table 3, changes in US GDP dynamics are quickly transmitted across both advanced and emerging economies. A unitary percentage change in US GDP leads in fact to a significant increase in median GDP for both OECD and non OECD countries at both horizons (0.16\% and $0.53 \%$, respectively, in the medium-term). ${ }^{23}$ By further grouping the countries in four groups (Tables 4 and 5), i.e. advanced Europe (plus Canada), Eastern Europe, Asia and Latin America, it is possible to note that the median medium-term responses for Europe and Eastern Europe are similar to those found for the OECD group (0.15\%), while for Asia and Latin America a stronger response is observed $(0.33 \%$ and $0.66 \%)$.

Overall, the economic slowdown in the US may be expected to have played a significant and sizable role in the worldwide economic recession, with a stronger effect for Latin American and Asian countries (South-Eastern Asia, especially) than for Eastern European and advanced economies, consistent with international trade linkages and own country growth dynamics. ${ }^{24}$ These findings are consistent with Dooley and Hutchinson (2009) and Levchenko et al. (2010), reporting a large decline in international trade (about 30\%$40 \%$ ) during the current crisis, and with Berken et al. (2009), Bems et al. (2010) and Grossman and Meissner (2010), pointing to the importance of the trade channel, particularly for countries exporting manufacturing and durable goods. Interestingly, our evidence actually contrasts with the decoupling of advanced and emerging economies business cycles hypothesis, recently put forward by Kose et al. (2008).

The US economic slowdown is also likely to have played a significant role in determining stock price developments in both advanced and emerging countries. In fact, Table 3 shows that similar median medium-term responses of foreign stock prices to US output dynamics is observed for both OECD (20\%) and non-OECD countries (27\%). Results for the European group are

\footnotetext{
${ }^{23}$ As can be noted in Table 3, in general, the dispersion of the cross-sectional distribution tends to be larger for non-OECD than OECD countries, revealing stronger commonalities in economic dynamics for the latter group of economies. Moreover, for both groups of countries and both horizons, the cross-sectional distribution features asymmetries and positive excess kurtosis, i.e. a larger number of outlying observations than compatible with a normal cross-sectional distribution.

${ }^{24}$ According to US Department of Commerce (http://www.census.gov/foreigntrade/Press-Release/current_press_release/ft900.pdf), in $200926 \%$ of total US imports was from North America (Canada 14\%, Mexico 11\%), 21\% from Europe (Germany 5\%, UK $3 \%$, France, Italy, Ireland $2 \%$ each, $7 \%$ other countries), $34 \%$ from Pacific Rim countries (19\% China, $6 \%$ Japan, $6 \%$ NICs, $4 \%$ other countries), South America 7\%, OPEC countries $7 \%$, other countries $5 \%$.
} 
again consistent with what found for the OECD group (22\%), while the Eastern European group shows a more muted reaction (11\%); on the other hand, stronger median responses are found for the Asian (25\%) and Latin American $(26 \%)$ areas, also consistent with the deeper impact of the US economic recession on foreign output for the latter groups of countries.

Finally, while for house prices the connection with US GDP dynamics is negligible, the response of excess credit is sizable and different across groups, i.e. positive for OECD countries (1.6\%) and negative for non-OECD countries $(-1.9 \%)$, and stronger for Eastern Europe $(+2.36 \%)$ and Latin America $(-1.65 \%)$ than for Europe $(1 \%)$ and Asia $(-0.5 \%)$. This pattern is possibly explained by a different monetary policy reaction across the two sub-groups of countries, being procyclical for OECD economies and countercyclical for non-OECD countries.

\subsection{Responses to US financial developments}

According to the results reported in Table 3, the effects of US financial developments on foreign output are not fully clear-cut. US stock price dynamics do not have any relevant effect on foreign GDP, while US house price dynamics do exercise some negative effects for the non-OECD group $(-0.22 \%$ in the medium-term); yet, a worsening of financial fragility conditions in the US leads to an output contraction for both groups (-0.12\%, OECD, short-term; $0.13 \%$, non OECD, medium-term). Moreover, the sub-group analysis reveals that US house price dynamics and financial fragility conditions are particularly relevant for Eastern Europe (-0.25\% and -0.89\%) and Latin America $(-0.30 \%$ and $-0.60 \%)$, leaving almost unaffected the European $(0.02 \%$ and $-0.03 \%)$ and Asian (0.04\% and $-0.1 \%$ ) groups.

Other interesting conclusions can be drawn for the foreign financial variables. First, US excess liquidity is positively associated with excess credit for OECD countries at both horizons $(0.2 \%$ to $0.5 \%)$, and house prices in the medium-term only $(0.17 \%)$; differently, the median impact on excess credit for non-OECD countries is negative ( $-0.66 \%$ in the medium-term). Consistent results are delivered by the sub-group analysis, pointing to sizable medium-term median contractions in excess credit for Eastern Europe $(-2.8 \%)$ and Latin America (-1.1\%), to a weaker response for Asia (-0.2\%), and to a positive response for Europe $(0.5 \%)$. Moreover, the effect of an increase in US excess liquidity on foreign stock prices is sizable and positive for both groups in the medium-term $(5 \%)$, but negative for non-OECD countries in the short-term $(-6 \%)$. Interestingly, the positive medium-term response found for non-OECD countries concerns Eastern Europe (3.1\%) and Latin America (6.6\%) only, as for Asia the response is still negative (-0.9\%). 
Also, the short-term negative response for non-OECD economies appears to be particularly strong for Asia and Latin America (-8\%), and much smaller for Eastern Europe (-1.2\%); rather, figures for Europe are in line with what found for the OECD group (3.2\% to $7.3 \%)$.

Second, US housing and stock prices do affect foreign financial markets of both group of countries. An increase in US house prices lead to a median increase in house prices for OECD countries, particularly in the mediumterm $(0.8 \%)$, and to an increase in stock prices for both OECD (16\% to 18\%) and non-OECD economies (9\% to $23 \%$ ); on the other hand, an increase in US stock prices causes an increase in stock prices in the OECD countries $(0.15 \%$ in the short-term), but a contraction in non-OECD stock markets $(-5 \%)$. Interestingly, the sub-group analysis reveals that while the positive effect of a US house price increase on international stock markets is similar in magnitude across sub-groups, the negative effect of a US stock prices increase would be larger for Latin American (-9\% to -11\%) than for Asian and Eastern European $(-2 \%$ to $-4 \%)$ countries.

It may then be concluded that a generous stance in US liquidity might lead to a rebalancing of international investor portfolios in favor of advanced and safer financial markets; hence, excess liquidity in the US, as well as buoyant US housing and stock markets, may have contributed to keep momentum in foreign advanced country stock and housing markets (and to their depression during the bust phase). Should the trend in liquidity creation be reversed, it may then be expected that stock markets in advanced economies would suffer more than those in emerging countries, as international investors appear to switch to emerging countries' stock markets when the US market stagnate or is depressed. This is also confirmed by the fact that a worsening of economic and financial fragility conditions in the US leads to a medium-term contraction in house $(-0.1 \%)$ and stock prices $(-5 \%)$ in OECD countries, but to an increase in stock prices in non-OECD economies $(14 \%$ in the short-term), particularly in Asia and Latin America (12\% and 5\%;-26\% for Eastern Europe). Overall, our findings are only partially consistent with Galesi and Sgherri (2009). Likewise the latter authors, we do find evidence of transmission of negative US stock price shocks to advanced and emerging European stock markets in the short-term; yet, we do also find that the effects of US shocks do not fade away in the short-term, still lasting also in the medium-term. 


\section{Conclusions}

The paper focuses on the current economic and financial turmoil, considering both its dynamics within the US and the spillover to foreign advanced and emerging economies. Relative to previous work in the literature, the current paper innovates for the depth and wideness of the analysis and econometric methodology, providing an accurate investigation of the international macro/finance interface. A total of 50 countries, covering advanced and major emerging countries, and 278 equations, considering key macroeconomic and financial variables, are in fact investigated by means of a large-scale open economy macroeconometric model, set in the factor vector autoregressive (FVAR) framework and estimated over the 1980-2009 period.

The main conclusions of the paper are as follows. First, concerning the mechanics of the crisis within the US, the empirical results are quite consistent with a boom-bust credit cycle interpretation of the crisis. In fact, there is evidence that asset prices misalignments in the housing and stock markets, as well as low real interest rates, over the boom phase of the cycle, might have been driven by excessively generous liquidity in the system. The ballooning US trade deficit also likely contributed to the latter dynamics, as huge capital inflows were redirected from the bond and stock markets to the housing market. Moreover, there is also evidence that the bust phase of the crisis may have been precipitated by declining house prices and the consequent breakdown in the predatory lending mechanism. The empirical evidence does in fact point to a bidirectional linkage relating house and stock prices, consistent with the generalized decline in asset prices and tight credit conditions, which resulted from deleveraging and recapitalization of financial institutions. In addition, concerning the real effects of the crisis, stronger evidence of an assets price channel, working through wealth and Tobin's "q" effects, than a liquidity channel, is detected. Also consistent is the related deflation risk, feared during the unwinding of the crisis, given the negative linkage between asset prices and inflation detected in the data. Moreover, concerning the policy reaction to the crisis, the evidence supports the effectiveness of the implemented expansionary fiscal/monetary policy mix, as deficit creation and real interest rate cuts both lead to a significant expansion in output and employment. Finally, the recession in the US may have been made worse by the second-round effects determined by weakened external demand, as foreign output is found to significantly affect US real activity, as well as US house and stock prices.

Second, concerning the spillovers of the crisis to foreign advanced and emerging economies, the contraction in real economic activity in the US may have played a sizable role in the slowdown of foreign economic activity, neg- 
atively affecting foreign financial markets as well. Interestingly, a stronger response is found for emerging economies, especially in Latin America and Asia, than for advanced economies, consistently with international trade linkages. On the other hand, adverse US financial developments do not seem to have a clear-cut impact on foreign economic activity. Hence, the trade channel does seem to be the key transmission mechanism of the US economic crisis to the rest of the world. Differently, the US financial crisis is likely to have spread through US house and stock price dynamics, as well as excess liquidity creation. 


\section{Appendix A: Identification of structural dis- turbances}

This Appendix describes the identification procedure used to extract structural disturbances from the reduced-form factor innovations in $\boldsymbol{\eta}_{t}$ and $\boldsymbol{\zeta}_{t}$, and from the vector of US-specific disturbances $\mathbf{v}_{t}^{X}$ to investigate the dynamic effects of various shocks within the US economy, starting from the reducedform $V M A$ representation of the US variables in $\mathbf{X}_{t}$ obtained from the whole system in (8):

$$
\mathbf{X}_{t}-\boldsymbol{\mu}_{t}^{X}=\mathbf{H}_{X F}(L) \boldsymbol{\eta}_{t}+\mathbf{H}_{X G}(L) \boldsymbol{\zeta}_{t}+\mathbf{H}_{X X}(L) \mathbf{v}_{t}^{X}
$$

where $\mathbf{H}_{X F}(L), \mathbf{H}_{X G}(L)$ and $\mathbf{H}_{X X}(L)$ are the appropriate lag polynomial sub-matrices of $\mathbf{H}(L)$ in (8). Structural disturbances are obtained by means of a standard identification scheme in structural VAR models based on exclusion restrictions on the contemporaneous (within quarter) responses of the elements in $\mathbf{F}_{t}, \mathbf{G}_{t}$ and $\mathbf{X}_{t}$ to shocks.

In particular, denoting by $\boldsymbol{\xi}_{t}$ the vector of the $r$ structural shocks driving the common factors in $\mathbf{F}_{t}$, the relation between the reduced form and the structural factor disturbances can be written as $\boldsymbol{\xi}_{t}=\mathbf{K} \boldsymbol{\eta}_{t}$, where $\mathbf{K}$ is a $r \times r$ invertible matrix. By assumption the structural factor shocks are orthogonal and have unit variance, so that $E\left[\boldsymbol{\xi}_{t} \boldsymbol{\xi}_{t}^{\prime}\right]=\mathbf{K} \boldsymbol{\Sigma}_{\eta} \mathbf{K}^{\prime}=\mathbf{I}_{r}$. To achieve exact identification of the structural disturbances, additional $r(r-1) / 2$ restrictions need to be imposed. Since $\boldsymbol{\eta}_{t}=\mathbf{K}^{-1} \boldsymbol{\xi}_{t}$, imposing exclusion restrictions on the contemporaneous impact matrix amounts to imposing zero restrictions on the elements of $\mathbf{K}^{-1}$, for which a lower-triangular structure is assumed. This latter assumption implies a precise "ordering" of the common factors in $\mathbf{F}_{t}$. In particular, the first factor is allowed to have a contemporaneous impact on all other factors, but reacts only with a one-period lag to the other structural disturbances; instead, the last factor is contemporaneously affected by all structural shocks, having only lagged effects on all other factors. Operationally, $\mathbf{K}^{-1}$ (with the $r(r-1) / 2$ zero restrictions necessary for exact identification imposed) is estimated by the Choleski decomposition of the factor innovation variance matrix $\boldsymbol{\Sigma}_{\eta}: \hat{\mathbf{K}}^{-1}=\operatorname{chol}\left(\hat{\boldsymbol{\Sigma}}_{\eta}\right)$.

A similar procedure is applied to obtain identification of the structural shocks driving the foreign (non-US) factors in $\mathbf{G}_{t}$ and the US-specific innovations in $\mathbf{v}_{t}^{X}$. Denoting by $\boldsymbol{\psi}_{t}$ the vector of $s$ structural shocks to the foreign factors (uncorrelated with the $\boldsymbol{\xi}_{t}$ shocks), the relation between the reduced form and the structural foreign factor disturbances can be written as $\boldsymbol{\psi}_{t}=\boldsymbol{\Gamma} \boldsymbol{\zeta}_{t}$, where $\boldsymbol{\Gamma}$ is a $s \times s$ invertible matrix. In addition to the orthogonality conditions $E\left[\boldsymbol{\psi}_{t} \boldsymbol{\psi}_{t}^{\prime}\right]=\boldsymbol{\Gamma} \boldsymbol{\Sigma}_{\psi} \boldsymbol{\Gamma}^{\prime}=\mathbf{I}_{s}, s(s-1) / 2$ zero restrictions are needed 
for exact identification. Similarly, denoting by $\boldsymbol{v}_{t}=\boldsymbol{\Theta} \mathbf{v}_{t}^{X}$, where $\boldsymbol{\Theta}$ is a $q \times q$ invertible matrix, the $q$-variate vector of US idiosyncratic structural shocks (uncorrelated with the above structural factor shocks), the identification of those disturbances requires, in addition to the orthogonality conditions $E\left[\boldsymbol{v}_{t}^{X} \boldsymbol{v}_{t}^{X \prime}\right]=\boldsymbol{\Theta} \boldsymbol{\Sigma}_{v^{X}} \boldsymbol{\Theta}^{\prime}=\mathbf{I}_{q}, q(q-1) / 2$ zero restrictions. Since $\boldsymbol{\zeta}_{t}=\boldsymbol{\Gamma}^{-1} \boldsymbol{\psi}_{t}$ and $\mathbf{v}_{t}^{X}=\Theta^{-1} \boldsymbol{v}_{t}$, the required restrictions are imposed by assuming a lowertriangular structure for the contemporaneous impact matrices $\Gamma^{-1}$ and $\Theta^{-1}$. Operationally, $\boldsymbol{\Gamma}^{-1}$ and $\boldsymbol{\Theta}^{-1}$ are estimated by the Choleski decomposition of the factor innovation variance matrix $\boldsymbol{\Sigma}_{\zeta}$ and the US idiosyncratic innovation variance matrix $\boldsymbol{\Sigma}_{v^{x}}: \hat{\boldsymbol{\Gamma}}^{-1}=\operatorname{chol}\left(\hat{\boldsymbol{\Sigma}}_{\zeta}\right)$ and $\boldsymbol{\Theta}^{-1}=\operatorname{chol}\left(\hat{\boldsymbol{\Sigma}}_{v^{x}}\right)$. The structural $V M A$ representation of the US variables in $\mathbf{X}_{t}$ then becomes:

$$
\mathbf{X}_{t}-\boldsymbol{\mu}_{t}^{X}=\mathbf{H}_{X F}(L) \mathbf{K}^{-1} \boldsymbol{\xi}_{t}+\mathbf{H}_{X G}(L) \boldsymbol{\Gamma}^{-1} \boldsymbol{\psi}_{t}+\mathbf{H}_{X X}(L) \boldsymbol{\Theta}^{-1} \boldsymbol{v}_{t}
$$

and is used to obtain the impulse response functions of US variables to common factor, foreign factor and US idiosyncratic structural disturbances. ${ }^{25}$

In our application, the assumption of uncorrelatedness among the factors in $\mathbf{F}_{t}$ is likely to apply already at the reduced form level, making the ordering of the structural shocks immaterial. Moreover, given the chosen univariate specification of the $\mathbf{G}_{t}$ process, the identification of the structural foreign (non-US) shocks does not require the computation of the Choleski decomposition. Finally, as to the effects of the country-specific shocks on the US series in $\mathbf{X}_{t}$, the ordering is likely to matter, and, as mentioned in the text, a suitable economic rationale is provided, based on the variables' speed of adjustment to shocks, with a distinction between relatively slow-moving (ordered first) and fast-moving variables (ordered last).

\footnotetext{
${ }^{25}$ Finally, in order to enforce orthogonality among structural shocks, prior to the computation of the $\boldsymbol{\Gamma}^{-1}$ and $\boldsymbol{\Theta}^{-1}$ matrices, the following steps are necessary: first, the foreignfactor F-VAR innovations $\hat{\zeta}_{t}$ estimated from (6) are regressed by OLS on the estimated structural common-factor disturbances $\hat{\boldsymbol{\xi}}_{t}$ to obtain a new estimate, $\tilde{\boldsymbol{\zeta}}_{t}$, from which the structural disturbances $\hat{\boldsymbol{\psi}}_{t}$ are then computed. Second, the US-specific reduced-form disturbances obtained from the F-VAR in (6), $\hat{\boldsymbol{\varepsilon}}_{t}^{X}$, are regressed on $\hat{\boldsymbol{\xi}}_{t}$ and $\hat{\boldsymbol{\psi}}_{t}$ by OLS to obtain an orthogonal estimate of the idiosyncratic disturbances, $\hat{\mathbf{v}}_{t}^{X}$, from which the structural disturbances $\hat{\boldsymbol{v}}_{t}$ are then computed.
} 


\section{References}

[1] Adalid, R. and C. Detken, 2007, Liquidity shocks and asset price boom/bust cycles, ECB Working Paper Series, no. 732.

[2] Alm, J. and J.R. Follain, 1984, Alternative mortgage instruments, the tilt problem, and consumer welfare, Journal of Financial and Quantitative Analysis, 19, 113-126.

[3] Almunia, M., A.S. Benetrix, B. Eichengreen, K.H. O'Rourke, and G. Rua, 2010, From Great Depression to Great Credit Crisis: Similarities, differences and lessons, Economic Policy, 62, 219-265.

[4] Bagliano, F.C. and C. Morana, 2008, Factor vector autoregressive estimation: a new approach, Journal of Economic Interaction and Coordination, 3, 15-23.

[5] Bagliano, F.C. and C. Morana, 2009, International macroeconomic dynamics: a factor vector autoregressive approach, Economic Modelling, $26,432-444$.

[6] Bai, J., 2003, Inferential theory for factor models of large dimensions, Econometrica, 71, 135-171.

[7] Barro R.J. and J.F. Ursua, 2009, Stock-market crashes and depressions, NBER Working Paper, no. 14760.

[8] Basurto, M.A.S., Goodhart, C. and B. Hoffman, 2006, Default, credit growth and asset prices, IMF Working Paper, no. 06/223.

[9] Bayoumi, T. and O. Melander, 2008, Credit matters: empirical evidence on U.S. macro-financial linkages, IMF Working Paper, no. 08/169.

[10] Becker, R., W. Enders and J. Lee, 2006, A stationarity test in the presence of an unknown number of smooth breaks, Journal of Time Series Analysis, 27, 381-409.

[11] Beltratti, A. and C. Morana, 2010, International house prices and macroeconomic fluctuations, Journal of Banking and Finance, 34, 535-545.

[12] Bems, R., L. Dedola and F. Smets, 2007, US imbalances - The role of technology and policy, Journal of International Money and Finance, 26, 523-545. 
[13] Bems, R., R.C. Johnson and K.-M. Yi, 2010, Demand spillovers and the collapse of trade during the Great Recession, IMF Working Paper, no. 10/142.

[14] Berken, P., G. Gelos, R. Rennhack, and J.P. Walsh, 2009, The global financial crisis: Explaining cross-country differences in the output impact, IMF Working Paper, no. 09/280.

[15] Bernanke, B., 1983, Non monetary effects of the financial crisis in the propagation mechanism of the Great Depression, American Economic Review, 73, 257-276.

[16] Bierens, H.J., 2000, Non parametric nonlinear cotrending analysis, with an application to interest and inflation in the United States, Journal of Business and Economic Statistics, 18, 323-37.

[17] Bordo, M.D. and H. James, 2009, The Great Depression analogy, NBER Working Paper, no.15584.

[18] Bordo, M.D. and J. Landon-Lane, 2010, The lessons from the banking panics in the United States in the 1930s and for the financial crisis of 2007-2008, NBER Working Paper, no.16365.

[19] Borio, C., 2008, The financial turmoil of 2007-?: A preliminary assessment and some policy considerations, BIS Working Paper, no. 251.

[20] Buiter, W.H., 2009, The limits to fiscal stimulus, CEPR Discussion Paper Series, no.7607.

[21] Carrol, C.D., M. Otsuka and J. Slacalek, 2006, How large is the housing wealth effect? A new approach, NBER Working Paper, no. 12746.

[22] Case K.E., J.M. Quigley, and R.J. Shiller, 2005, Comparing wealth effects: The stock market versus the housing market, Advances in Macroeconomics, 5, art.1, 1-33.

[23] Cecchetti, S.G., 2006, Measuring the macroeconomic risks posed by asset price booms, NBER Working Paper, no.12542.

[24] Cecchetti, S.G., 2009, Financial crisis and economic activity, 2009, CEPR Discussion Paper Series, no.7495.

[25] Chirinko R.S., L.de Han and E. Sterken, 2004, Asset price shocks, real expenditures, and financial structure: a multicountry analysis, De Nederlansche Bank, Working Paper, no. 14/04. 
[26] Claessens, S., Kose M.A., and M.E. Terrones, 2009, What happens during recessions, crunches and busts?, Economic Policy, 60, 653-700.

[27] Dell'Ariccia, G., Detragiache, E. and R. Rajan, 2008, The real effects of banking crises, Journal of Financial Intermediation, 17, 89-112.

[28] Dees, S., M.H. Pesaran, L.V. Smith, and R.P. Smith, 2010, Supply, demand and monetary policy shocks in a multi-country New Keynesian model, ECB Working Paper Series, no. 1239. Available at http://www.ecb.int/pub/pdf/scpwps/ecbwp1239.pdf.

[29] Dooley and Hutchinson, 2009, Transmission of the U.S. subprime crisis to emerging markets: Evidence on the decoupling-recoupling hypothesis, Journal of International Money and Finance, 28, 1331-1349.

[30] Eichengreen, B. and K. Mitchener, 2004, The Great Depression as a credit boom gone wrong, Research in Economic History, 22, 183-237.

[31] Fratzscher, M., L. Juvenal, and L. Sarno, 2009, Asset prices, exchange rates and the current account, Federal Reserve Bank of St. Louis Working Paper, no.2008-031B, forthcoming European Economic Review.

[32] Galesi A, and S. Sgherri, 2009, Regional financial spillovers across Europe: A global VAR analysis, IMF Working Paper, no. 09/23.

[33] Gallant, R., 1984, The Fourier flexible form, American Journal of Agicultural Economics, 66, 204-08.

[34] Gauger, J. and T.C. Snyder, 2003, Residential fixed investment and the macroeconomy: Has deregulation altered key relationships?, Journal of Real Estate, Finance and Economics, 27, 335-354.

[35] Goodhart, C. and B. Hoffman, 2008, House prices, money, credit and the macroeconomy, Oxford Review of Economic Policy, 24, 180-205.

[36] Gordon, R.J., 2005, What caused the decline in U.S. business cycle volatility?, NBER Working Paper, no. 11777.

[37] Granger, C.W. and Y. Jeon, 2004, Thick modelling, Economic Modelling, 21, 323-43.

[38] Greenlaw, D., Hatzius, J.,. Kashyap, A.K. and H.S. Shin, 2008, Leveraged losses: Lessons from the mortgage market meltdown, Chicago University, mimeo, available at http://www.brandeis.edu/global/rosenberg_institute/usmpf_2008.pdf. 
[39] Grossman, R.S. and C.M. Meissner, 2010, International aspects of the Great Depression and the crisis of 2007: Similarities, differences and lessons, NBER Working Paper, no. 16269.

[40] Hamilton, J., 1996, This is What Happened to the Oil PriceMacroeconomy Relationship, Journal of Monetary Economics, 38, 21520.

[41] Jagannathan, R., Kapoor M., and E. Schaumburg, 2009, Why are we in a recession? The financial crisis is the symptom not the disease!, NBER Working Paper n.15404.

[42] Kose, M.A., Otrok, C. and E. Prasad, 2008, Global business cycles: Convergence or decoupling?, NBER Working Paper, no. 14292.

[43] Kwiatkowski, D., P.C.B. Phillips, P. Schmidt and Y. Shin, 1992, Testing the null hypothesis of stationarity against the alternative of a unit root, Journal of Econometrics, 54, 159-78.

[44] Leamer, E., 2007, Housing is the business cycle, NBER Working Paper, no. 13428 .

[45] Levchenko, A.A., Lewis, L.T. and L.L. Tesar, 2010, The collapse of international trade during the 2008-2009 crisis: In search for the smoking gun, NBER Working Paper, no.16006.

[46] Levine, R., 2010, An autopsy of the US financial system, NBER Working Paper, no.15956.

[47] Morana, C, 2005, The Japanese deflation: Has it had real effects? Could it have been avoided?, Applied Economics, 37, 1337-52.

[48] Morana, C., 2006, A small scale macroeconometric model for the euro-12 area, Economic Modelling, 23, 391-426.

[49] Pesaran, M.H. and A. Chudik, 2010, Econometric analysis of high dimensional VARs featuring a dominant unit, ECB Working Paper Series, no. 1194. Available at http://www.ecb.int/pub/pdf/scpwps/ecbwp1194.pdf.

[50] Reinhart, C. and K.S. Rogoff, 2009a, The aftermath of financial crises, American Economic Review, 99, 2, 466-472.

[51] Reinhart, C. and K.S. Rogoff, 2009b, This time is different. Eight centuries of financial folly, Princeton, Princeton University Press. 
[52] Reis, R., 2010, Interpreting the unconventional US monetary policy of 2007-09, mimeo, Columbia University, available at http://papers.ssrn.com/sol3/papers.cfm?abstract_id=1539307.

[53] Shularick, M. and A.M. Taylor, 2009, Credit booms gone bust: Monetary policy, leverage, cycles and financial crises, 1870-2008, NBER Working Paper, no.15512.

[54] Stock, J.H. and M.W. Watson, 2005, Implications of dynamic factor models for VAR analysis, NBER Working Paper, no. 11467.

[55] Temin, P., 2010, The Great Recession and the Great Depression, NBER Working Paper, no. 15645. 


\section{Figure 1}

(a) US financial fragility index

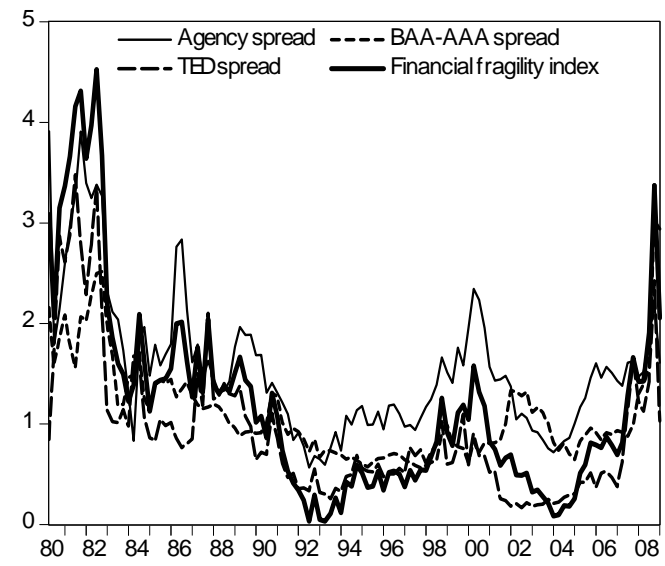

(b) US excess liquidity index

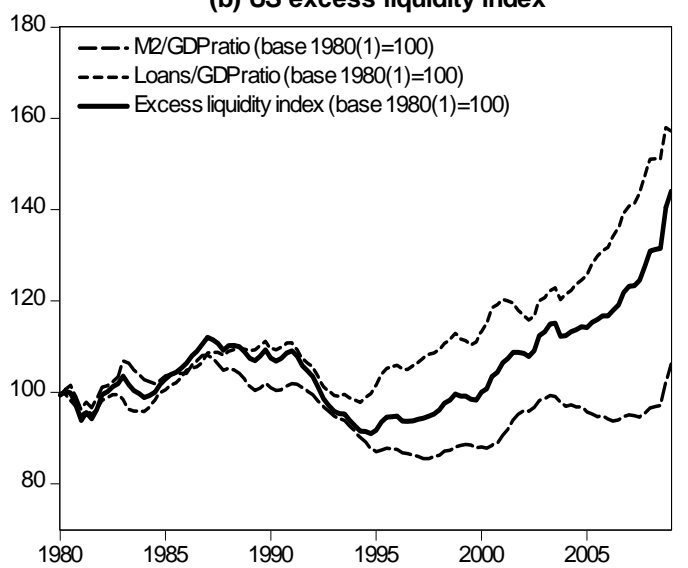

(c) Common non-US GDP growth rate factor

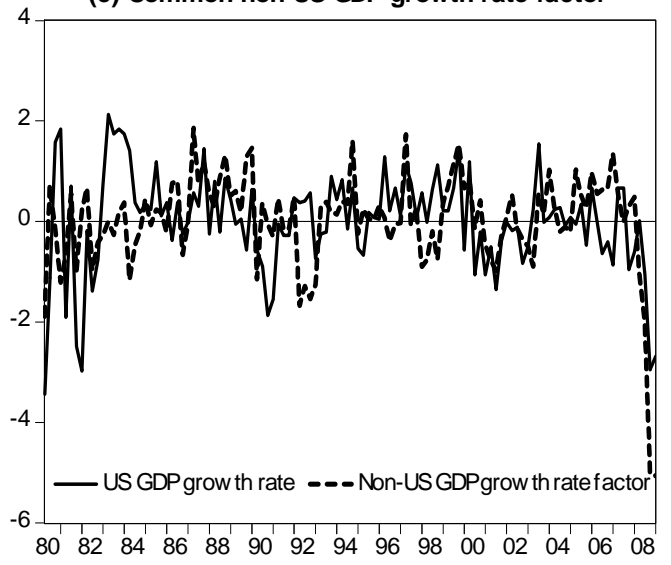

Panel (a) shows the US financial fragility index and the three spread series (Agency, BAA-AAA, and TED); panel (b) plots the US M2 to GDP ratio, Bank loans to GDP ratio (both in index form) and the extracted US excess liquidity index; panel (c) portrays the (standardized) non_US common GDP growth factor together with the US GDP growth rate. The sample is: 1980:1-2009:1. 
Table 1: Forecast error variance decomposition for US variables

\begin{tabular}{|c|c|c|c|c|c|c|c|c|c|c|c|c|c|c|}
\hline \multirow[b]{2}{*}{ resp $\backslash$ sh } & \multicolumn{14}{|c|}{ Panel A: 2-quarter horizon } \\
\hline & $e$ & $g$ & $p d$ & $c$ & $i$ & cad & $\pi$ & exl & $s$ & $l$ & $h$ & $e r$ & $f$ & $f r$ \\
\hline$e$ & 90.7 & 6.8 & 0.16 & 0.03 & 0.25 & 0.20 & 0.30 & 0.22 & 0.21 & 0.30 & 0.09 & 0.14 & 0.09 & 0.51 \\
\hline$g$ & 23.1 & 68.3 & 0.11 & 1.33 & 0.06 & 0.00 & 5.28 & 0.00 & 0.61 & 0.01 & 0.31 & 0.18 & 0.24 & 0.47 \\
\hline$p d$ & 4.81 & 1.18 & 79.5 & 0.42 & 0.53 & 1.86 & 0.93 & 1.79 & 1.30 & 0.25 & 4.59 & 0.77 & 1.75 & 0.33 \\
\hline$c$ & 19.0 & 18.4 & 2.02 & 54.6 & 0.22 & 0.93 & 0.75 & 0.00 & 0.71 & 0.12 & 2.08 & 0.00 & 0.08 & 1.06 \\
\hline$i$ & 26.7 & 27.4 & 0.11 & 6.57 & 32.4 & 0.10 & 0.49 & 0.34 & 3.01 & 0.17 & 0.48 & 0.13 & 0.91 & 1.00 \\
\hline cad & 1.28 & 1.23 & 1.57 & 0.03 & 0.24 & 88.0 & 0.73 & 0.01 & 2.27 & 0.23 & 0.18 & 0.06 & 3.84 & 0.31 \\
\hline$\pi$ & 0.41 & 0.44 & 1.02 & 27.7 & 0.10 & 3.14 & 64.2 & 0.25 & 1.45 & 0.04 & 0.71 & 0.28 & 0.16 & 0.07 \\
\hline exl & 2.17 & 37.7 & 0.19 & 10.8 & 4.50 & 1.07 & 3.69 & 37.7 & 0.10 & 0.86 & 0.27 & 0.43 & 0.43 & 0.02 \\
\hline$s$ & 6.56 & 0.66 & 4.14 & 16.7 & 0.61 & 2.03 & 47.4 & 1.19 & 19.2 & 0.09 & 1.14 & 0.25 & 0.02 & 0.02 \\
\hline$l$ & 2.39 & 1.07 & 6.11 & 21.4 & 0.59 & 0.43 & 53.8 & 0.77 & 4.01 & 7.14 & 1.69 & 0.20 & 0.25 & 0.17 \\
\hline$h$ & 1.13 & 1.13 & 1.66 & 14.9 & 1.21 & 1.60 & 29.2 & 0.33 & 1.42 & 0.17 & 46.9 & 0.00 & 0.04 & 0.28 \\
\hline$e r$ & 1.66 & 1.47 & 0.60 & 1.46 & 1.66 & 0.64 & 0.20 & 0.09 & 2.92 & 3.13 & 0.66 & 84.7 & 0.80 & 0.01 \\
\hline$f$ & 0.17 & 1.32 & 5.63 & 4.38 & 0.92 & 11.1 & 6.22 & 1.62 & 1.80 & 13.7 & 2.20 & 2.95 & 47.8 & 0.21 \\
\hline$f r$ & 3.56 & 0.08 & 2.04 & 3.70 & 1.66 & 1.58 & 14.1 & 2.55 & 9.05 & 2.22 & 1.91 & 1.06 & 4.83 & 51.7 \\
\hline
\end{tabular}

\begin{tabular}{|c|c|c|c|c|c|c|c|c|c|c|c|c|c|c|}
\hline & \multicolumn{14}{|c|}{ Panel C: 12-quarter horizon } \\
\hline resp $\backslash$ sh & $e$ & $g$ & $p d$ & $c$ & $i$ & cad & $\pi$ & exl & $s$ & $l$ & $h$ & er & $f$ & fr \\
\hline$e$ & 47.7 & 13.7 & 2.27 & 0.81 & 1.37 & 1.10 & 7.59 & 0.43 & 17.1 & 3.26 & 2.57 & 0.20 & 1.57 & 0.26 \\
\hline$g$ & 11.0 & 44.8 & 3.09 & 0.35 & 1.62 & 2.76 & 11.5 & 0.26 & 18.3 & 3.68 & 0.76 & 0.05 & 1.45 & 0.35 \\
\hline$p d$ & 6.68 & 1.18 & 64.7 & 0.96 & 0.57 & 5.64 & 0.94 & 1.52 & 5.06 & 2.71 & 6.52 & 0.82 & 2.06 & 0.61 \\
\hline$c$ & 13.3 & 13.4 & 2.80 & 38.3 & 0.22 & 12.8 & 1.19 & 0.38 & 0.30 & 1.39 & 8.22 & 0.01 & 0.27 & 7.38 \\
\hline$i$ & 15.2 & 24.2 & 0.53 & 2.81 & 11.9 & 12.9 & 1.76 & 0.06 & 21.2 & 1.87 & 6.08 & 0.04 & 1.00 & 0.43 \\
\hline cad & 1.39 & 4.65 & 1.08 & 0.78 & 0.45 & 44.0 & 4.03 & 0.03 & 15.5 & 12.6 & 6.51 & 0.40 & 6.07 & 2.51 \\
\hline$\pi$ & 2.74 & 0.21 & 4.80 & 22.7 & 0.79 & 8.61 & 41.2 & 0.28 & 9.04 & 3.65 & 3.71 & 0.74 & 1.45 & 0.07 \\
\hline exl & 3.74 & 13.7 & 1.33 & 6.64 & 5.25 & 20.3 & 0.59 & 32.9 & 9.34 & 1.52 & 1.26 & 0.36 & 0.14 & 2.98 \\
\hline$s$ & 7.41 & 2.32 & 4.07 & 14.5 & 0.91 & 2.58 & 40.3 & 1.68 & 19.9 & 0.67 & 1.42 & 0.50 & 1.09 & 2.68 \\
\hline$l$ & 2.61 & 2.91 & 7.00 & $\begin{array}{ll}17.3 \\
\end{array}$ & 0.84 & 1.12 & 44.9 & 1.21 & 4.88 & 11.8 & 2.60 & 0.36 & 1.56 & 0.91 \\
\hline$h$ & 2.05 & 0.64 & 1.12 & 12.4 & 0.12 & 0.36 & 15.1 & 0.84 & 11.1 & 8.96 & 41.8 & 0.47 & 3.14 & 1.98 \\
\hline$e r$ & 4.83 & 0.56 & 0.94 & 4.36 & 3.28 & 8.85 & 1.92 & 0.17 & 8.63 & 1.12 & 1.20 & 61.7 & 2.11 & 0.35 \\
\hline$f$ & 0.20 & 0.80 & 1.62 & 1.51 & 0.55 & 19.5 & 9.81 & 0.29 & 1.37 & 8.42 & 2.62 & 3.53 & 49.6 & 0.20 \\
\hline$f r$ & 6.82 & 0.18 & 5.41 & 7.11 & 2.97 & 0.87 & 21.1 & 2.10 & 16.7 & 1.98 & 2.89 & 1.26 & 3.21 & 27.4 \\
\hline
\end{tabular}

The Table reports the results of the forecast error variance decomposition analysis for the US variables (rows), relative to the US shocks (columns). For instance element $(1,2)$ in Panel A, i.e. 6.8, is the percentage of forecast error variance of US employment explained by the US output shock. The variables are real GDP $(g)$, civilian employment $(e)$, real private consumption $(c)$, real private investment $(i)$, fiscal deficit to GDP $(p d)$, current account deficit to GDP $(c a d)$, CPI all items index $(\pi)$, three-month Treasury Bills real rate $(s)$, 10-year Federal government securities real rate $(l)$, real house prices $(h)$, the real effective exchange rate $(e r)$, real share prices $(f)$, the economic/financial fragility indexed $(f r)$, and the excess liquidity index $(\mathrm{exl})$. 
Table 2: Median cumulated impulse response analysis for US variables (selected shocks)

\begin{tabular}{|c|c|c|c|c|c|c|c|c|c|c|c|c|c|c|c|}
\hline $\operatorname{sh} \downarrow$ & Resp $\rightarrow$ & $e$ & $g$ & $p d$ & $c$ & $i$ & cad & $\pi$ & exl & $s$ & $l$ & $\boldsymbol{h}$ & $e r$ & $f$ & $f r$ \\
\hline & 2 & 0.32 & 0.31 & -0.08 & 0.24 & 0.79 & 0.05 & 0.04 & -0.12 & 0.09 & -0.11 & -0.02 & 0.34 & 0.28 & 0.00 \\
\hline \multirow[t]{3}{*}{$e$} & 4 & 0.33 & 0.30 & -0.03 & 0.23 & 0.99 & 0.05 & 0.12 & -0.03 & 0.19 & 0.08 & -0.10 & 0.43 & 0.00 & 0.05 \\
\hline & 12 & 0.25 & 0.18 & 0.00 & 0.24 & 0.50 & -0.01 & 0.15 & 0.22 & 0.05 & 0.04 & -0.22 & 0.43 & -0.08 & 0.02 \\
\hline & 2 & 0.11 & 0.43 & 0.05 & 0.22 & 0.85 & 0.04 & 0.03 & -0.43 & 0.10 & 0.17 & -0.10 & -0.25 & 0.65 & -0.01 \\
\hline \multirow[t]{4}{*}{$g$} & 4 & 0.15 & 0.48 & 0.00 & 0.21 & 0.92 & 0.06 & -0.01 & -0.35 & 0.10 & 0.14 & -0.01 & 0.01 & 0.15 & 0.02 \\
\hline & 12 & 0.18 & 0.50 & 0.01 & 0.23 & 0.91 & 0.05 & -0.07 & -0.16 & 0.00 & 0.00 & 0.17 & -0.23 & 0.68 & 0.00 \\
\hline & & & & & & & & & & & & & & & \\
\hline & 2 & 0.02 & 0.03 & 0.06 & -0.08 & -0.05 & -0.02 & -0.07 & -0.03 & 0.24 & 0.40 & -0.06 & 0.18 & -1.29 & 0.01 \\
\hline \multirow[t]{2}{*}{$p d$} & 4 & 0.02 & 0.07 & 0.02 & -0.10 & 0.00 & -0.02 & -0.13 & -0.05 & 0.09 & 0.16 & 0.03 & -0.08 & -0.90 & -0.05 \\
\hline & 12 & 0.10 & 0.19 & 0.00 & -0.12 & 0.26 & 0.02 & -0.24 & -0.15 & -0.03 & -0.03 & 0.20 & -0.32 & 0.21 & -0.03 \\
\hline & 2 & ?ח0 & $0 \Omega 0$ & 006 & & $0 \Omega$ & 026 & 012 & 007 & O & 008 & 011 & (1) & 104 & \\
\hline \multirow{2}{*}{ cad } & 4 & -0.01 & -0.06 & 0.04 & -0.18 & -0.38 & 0.19 & -0.22 & 0.27 & -0.07 & -0.02 & -0.12 & -0.17 & 2.67 & 0.02 \\
\hline & 12 & -0.06 & -0.16 & 0.00 & -0.28 & -0.84 & 0.04 & -0.28 & 0.44 & -0.02 & -0.04 & 0.05 & -0.84 & -2.67 & 0.00 \\
\hline & & & & & & & & & & & & & & & \\
\hline & 2 & -0.02 & -0.17 & 0.04 & -0.06 & -0.13 & -0.04 & 0.46 & -0.06 & -0.19 & -0.19 & -0.43 & -0.10 & -1.53 & 0.14 \\
\hline \multirow[t]{2}{*}{$\pi$} & 4 & -0.11 & -0.25 & 0.02 & -0.01 & -0.22 & -0.07 & 0.51 & -0.03 & 0.09 & 0.05 & -0.45 & 0.04 & -1.77 & 0.11 \\
\hline & 12 & -0.12 & -0.24 & 0.00 & 0.12 & -0.24 & -0.01 & 0.56 & 0.06 & 0.09 & 0.10 & -0.51 & 0.43 & -1.57 & 0.03 \\
\hline & 2 & $00 ?$ & 000 & -006 & 000 & -011 & 000 & 004 & 0.41 & -013 & -014 & -006 & 006 & -060 & 0.05 \\
\hline \multirow[t]{4}{*}{ exl } & 4 & 0.02 & 0.05 & 0.01 & 0.04 & 0.08 & 0.00 & 0.06 & 0.41 & 0.10 & 0.07 & -0.12 & 0.04 & 0.21 & 0.04 \\
\hline & 12 & 0.03 & 0.05 & 0.00 & 0.05 & -0.01 & 0.00 & 0.03 & 0.49 & 0.02 & 0.02 & -0.11 & 0.06 & 0.15 & 0.00 \\
\hline & & & & & & & & & & & & & & & \\
\hline & 2 & -0.02 & -0.06 & -0.05 & -0.06 & -0.33 & $\mathbf{- 0 . 0 7}$ & 0.09 & 0.03 & 0.19 & -0.13 & -0.13 & 0.33 & -0.90 & 0.11 \\
\hline \multirow[t]{2}{*}{$\boldsymbol{s}$} & 4 & -0.12 & -0.27 & -0.09 & -0.04 & -0.70 & -0.13 & 0.21 & 0.27 & 0.17 & 0.00 & -0.20 & 0.42 & -0.51 & 0.10 \\
\hline & 12 & -0.22 & -0.34 & 0.00 & 0.05 & -0.98 & -0.07 & 0.30 & 0.29 & 0.06 & 0.08 & -0.61 & 0.86 & -0.68 & 0.03 \\
\hline & 2 & 0.01 & 0.04 & -0.10 & 0.10 & 0.13 & 0.02 & 0.06 & -0.05 & -0.17 & -0.22 & 0.62 & -0.16 & 1.08 & -0.04 \\
\hline \multirow[t]{2}{*}{$\boldsymbol{h}$} & 4 & 0.09 & 0.09 & -0.05 & 0.20 & 0.60 & 0.09 & 0.12 & -0.14 & 0.08 & -0.05 & 0.79 & -0.28 & 1.31 & -0.01 \\
\hline & 12 & 0.03 & -0.07 & 0.00 & 0.19 & 0.23 & 0.03 & 0.20 & 0.05 & 0.02 & -0.01 & 0.87 & -0.28 & 0.66 & 0.03 \\
\hline & & & & & & & & & & & & & & & \\
\hline & 2 & 0.01 & 0.04 & 0.06 & -0.02 & 0.18 & 0.09 & -0.03 & -0.06 & 0.02 & 0.08 & 0.02 & 0.24 & 3.47 & -0.03 \\
\hline \multirow[t]{3}{*}{$f$} & 4 & 0.02 & $\mathbf{0 . 0 8}$ & 0.01 & 0.00 & 0.10 & 0.08 & -0.08 & -0.02 & -0.01 & 0.00 & 0.16 & -0.11 & 3.69 & -0.04 \\
\hline & 12 & 0.06 & 0.07 & 0.00 & -0.06 & 0.13 & 0.03 & -0.12 & 0.01 & -0.02 & -0.03 & 0.29 & -0.42 & 3.85 & 0.00 \\
\hline & 2 & -0.03 & -0.05 & 0.03 & 0.07 & -0.19 & 0.03 & -0.02 & 0.01 & 0.02 & 0.07 & 0.06 & 0.03 & 0.33 & 0.17 \\
\hline \multirow[t]{2}{*}{$f r$} & 4 & 0.01 & 0.06 & 0.00 & 0.15 & 0.11 & 0.06 & -0.04 & 0.00 & 0.06 & -0.01 & 0.17 & 0.02 & 0.34 & 0.08 \\
\hline & 12 & -0.01 & -0.03 & 0.01 & $\begin{array}{l}0.21 \\
\end{array}$ & -0.12 & 0.02 & 0.01 & 0.26 & 0.05 & 0.04 & 0.20 & 0.12 & -0.11 & 0.03 \\
\hline & & & & & & & & & & & & & & & \\
\hline & 2 & 0.00 & -0.15 & -0.08 & 0.31 & 0.33 & 0.21 & 0.65 & 0.01 & -2.86 & -3.10 & -1.30 & -5.35 & -12.0 & 0.32 \\
\hline \multirow[t]{2}{*}{$\boldsymbol{o}$} & 4 & -0.17 & -0.43 & 0.02 & -0.01 & -0.68 & -0.33 & 0.81 & 0.49 & -0.58 & -0.68 & -2.38 & -6.20 & -12.1 & 0.38 \\
\hline & 12 & -0.52 & -0.77 & -0.01 & -0.02 & -1.22 & -0.25 & 1.07 & 0.12 & 0.13 & 0.20 & -3.64 & -3.43 & -17.6 & 0.06 \\
\hline & 2 & 0.01 & 0.39 & -0.54 & 0.43 & -1.86 & -0.16 & 0.31 & -1.24 & -1.65 & -1.59 & -1.66 & -9.60 & -85.5 & 1.95 \\
\hline \multirow[t]{3}{*}{$c p$} & 4 & -0.27 & -0.41 & -0.73 & 0.61 & -3.65 & -1.80 & 1.78 & -0.07 & -2.60 & -2.90 & -5.05 & -12.9 & -84.9 & 0.65 \\
\hline & 12 & -1.23 & -1.37 & -0.05 & 1.14 & -4.94 & -0.81 & 2.36 & -1.11 & 0.40 & 0.50 & -8.75 & -0.79 & -91.9 & 0.13 \\
\hline & 2 & 0.03 & 0.07 & -0.04 & 0.09 & 0.32 & -0.01 & 0.05 & -0.10 & -0.08 & -0.12 & -0.02 & -0.51 & 4.50 & -0.13 \\
\hline \multirow[t]{2}{*}{$g f$} & 4 & 0.04 & 0.10 & 0.03 & 0.04 & 0.38 & $\mathbf{0 . 0 7}$ & -0.05 & -0.13 & 0.17 & 0.20 & 0.08 & -0.16 & 3.56 & 0.03 \\
\hline & 12 & 0.07 & 0.09 & 0.00 & 0.03 & 0.38 & 0.03 & -0.02 & -0.05 & -0.01 & -0.03 & 0.16 & -0.59 & 3.78 & 0.00 \\
\hline
\end{tabular}

The Table reports the results of the median cumulated impulse response analysis for the US variables (columns), relative to the various shocks (rows). The variables are real GDP $(g)$, civilian employment $(e)$, real private consumption $(c)$, real private investment $(i)$, fiscal deficit to GDP $(p d)$, current account deficit to GDP $(c a d)$, CPI all items index $(\pi)$, three-month Treasury Bills real rate $(s)$, 10-year Federal government securities real rate $(l)$, real house prices $(h)$, real share prices $(f)$, the economic/financial fragility index $(f r)$, the excess liquidity index $(e x l)$, the oil price $(o)$, the ex-energy commodity price index $(c p)$, and foreign output $(g f)$. Figures in bold are significant at the $10 \%$ level. 
Table 3: Median cumulated response of (selected) foreign variables to US shocks for OECD and non OECD countries

\begin{tabular}{|c|c|c|c|c|c|c|c|c|c|c|c|c|c|c|c|c|c|c|c|c|}
\hline \multicolumn{21}{|c|}{ Response of foreign output to unitary US shocks } \\
\hline & \multicolumn{4}{|c|}{ output shock } & \multicolumn{4}{|c|}{ excess liquidity shock } & \multicolumn{4}{|c|}{ house price shock } & \multicolumn{4}{|c|}{ stock price shock } & \multicolumn{4}{|c|}{ fragility shock } \\
\hline & \multicolumn{2}{|c|}{ OECD } & \multicolumn{2}{|c|}{ non OECD } & \multicolumn{2}{|c|}{ OECD } & \multicolumn{2}{|c|}{ non OECD } & \multicolumn{2}{|c|}{ OECD } & \multicolumn{2}{|c|}{ non OECD } & \multicolumn{2}{|c|}{ OECD } & \multicolumn{2}{|c|}{ non OECD } & \multicolumn{2}{|c|}{ OECD } & \multicolumn{2}{|c|}{ non OECD } \\
\hline & 2 & 12 & 2 & 12 & 2 & 12 & 2 & 12 & 2 & 12 & 2 & 12 & 2 & 12 & & 12 & 2 & 12 & & 12 \\
\hline Mean & 0.25 & 0.27 & 0.96 & 1.58 & -0.07 & -0.03 & 0.15 & 0.54 & -0.01 & 0.03 & -0.20 & -0.32 & 0.00 & 0.00 & 0.07 & 0.02 & -0.18 & -0.15 & $\begin{array}{l}-0.53 \\
\end{array}$ & $\begin{array}{l}-0.99 \\
\end{array}$ \\
\hline Std & 0.37 & 0.33 & 1.75 & 2.78 & 0.28 & 0.32 & 2.03 & 2.03 & 0.50 & 0.37 & 1.86 & 2.02 & 0.05 & 0.05 & 0.26 & 0.23 & 0.91 & 0.67 & 8.10 & 8.29 \\
\hline Q1 & 0.03 & 0.06 & 0.21 & 0.12 & -0.09 & -0.05 & -0.31 & -0.42 & -0.09 & -0.08 & -1.05 & -1.07 & -0.01 & -0.01 & -0.02 & -0.05 & -0.53 & -0.32 & -1.10 & -1.40 \\
\hline Median & 0.12 & 0.16 & 0.49 & 0.53 & -0.01 & 0.01 & -0.02 & 0.01 & 0.01 & 0.02 & -0.10 & -0.22 & 0.00 & 0.00 & 0.01 & 0.00 & -0.12 & -0.06 & 0.09 & -0.13 \\
\hline Q3 & 0.29 & 0.39 & 1.38 & 2.31 & 0.05 & 0.08 & 0.53 & 0.71 & 0.07 & 0.10 & 0.24 & -0.01 & 0.01 & 0.01 & 0.09 & 0.05 & 0.02 & 0.02 & 0.83 & 0.39 \\
\hline Sk & 2.19 & 1.41 & 1.89 & 2.25 & -1.04 & -2.35 & -0.94 & 0.58 & -2.43 & -0.33 & 0.06 & 0.34 & -0.67 & 0.39 & 0.96 & -0.98 & -0.88 & -2.28 & -1.39 & -1.08 \\
\hline $\mathrm{Ku}$ & 8.36 & 5.76 & 6.99 & 8.15 & 5.20 & 12.61 & 5.58 & 2.98 & 14.69 & 8.62 & 3.41 & $\begin{array}{l}4.66 \\
\end{array}$ & 6.76 & 6.06 & 5.31 & 5.69 & 9.11 & 11.92 & 8.06 & 7.49 \\
\hline \multicolumn{21}{|c|}{ Response of foreign excess credit to unitary US shocks } \\
\hline & & outpu & shock & & & cess liq & dity sho & & & louse pr & ce shocl & & & stock pr & ce shoc & & & fragilit & shock & \\
\hline & $\mathbf{O E}$ & & non 0 & CD & & CD & non $\mathrm{OF}$ & & $\mathbf{O E}$ & CD & non 0 & CD & $\mathrm{OF}$ & CD & non 0 & ECD & $\mathbf{O E}$ & & non $\mathrm{Ol}$ & CD \\
\hline & 2 & 12 & 2 & 12 & 2 & 12 & 2 & 12 & 2 & 12 & 2 & 12 & 2 & 12 & 2 & 12 & 2 & 12 & 2 & 12 \\
\hline Mean & 3.09 & 5.25 & -3.22 & $\begin{array}{l}-3.16 \\
\end{array}$ & 0.59 & 0.11 & -0.58 & -1.82 & 0.13 & 0.28 & -0.95 & $\begin{array}{l}-1.86 \\
\end{array}$ & \begin{tabular}{|c|}
-0.03 \\
\end{tabular} & $\begin{array}{l}-0.09 \\
\end{array}$ & -0.22 & 0.01 & 2.34 & 2.74 & 1.41 & 0.80 \\
\hline Std & 6.30 & 10.37 & 6.63 & 7.08 & 2.51 & 4.01 & 6.19 & 7.79 & 2.60 & 2.25 & 6.90 & 7.61 & 0.65 & 0.77 & 1.83 & 1.74 & 5.80 & 4.45 & 15.80 & 13.56 \\
\hline Q1 & -0.35 & -0.01 & -3.69 & -5.64 & -0.27 & -0.27 & -1.81 & -4.59 & -0.55 & -0.27 & -1.18 & -1.64 & -0.09 & -0.09 & -0.27 & -0.15 & -0.06 & 0.11 & -7.21 & -5.16 \\
\hline Median & 0.55 & 1.61 & -0.67 & -1.87 & 0.23 & 0.51 & 0.07 & -0.66 & -0.01 & 0.08 & $\begin{array}{l}-0.09 \\
\end{array}$ & -0.07 & 0.00 & 0.00 & -0.10 & -0.05 & 0.59 & 0.82 & -1.30 & -0.43 \\
\hline Q3 & 3.84 & 5.29 & -0.22 & 0.07 & 0.88 & 1.70 & 1.42 & 1.48 & 0.92 & 0.86 & 1.63 & 1.48 & 0.09 & 0.16 & 0.08 & 0.17 & 2.10 & 3.10 & 7.36 & 3.32 \\
\hline Sk & 2.66 & 3.17 & -2.50 & -1.62 & -1.28 & -2.23 & -2.27 & -1.98 & -0.28 & -0.06 & -2.95 & -2.30 & -1.55 & -2.68 & -2.08 & -0.66 & 1.27 & 1.92 & 1.33 & 1.31 \\
\hline $\mathrm{Ku}$ & 10.75 & 14.33 & 8.93 & 6.13 & 8.56 & 8.87 & 9.12 & 7.43 & 11.10 & 5.99 & 11.31 & 7.56 & 8.36 & 12.29 & 9.13 & 7.15 & 5.00 & 5.78 & 6.32 & 6.44 \\
\hline & & & & & & & & sponse $\mathrm{a}$ & eign ho & se price & to unit & ryUS sl & & & & & & & & \\
\hline & 2 & 12 & 2 & 12 & 2 & 12 & 2 & 12 & 2 & 12 & 2 & 12 & 2 & 12 & 2 & 12 & 2 & 12 & 2 & 12 \\
\hline Mean & 0.09 & -0.04 & & & -0.15 & -0.06 & & & 0.43 & 0.94 & & & 0.01 & $\begin{array}{l}-0.01 \\
\end{array}$ & & & 0.33 & -0.05 & & \\
\hline Std & 0.55 & 1.29 & & & 0.26 & 0.86 & & & 0.67 & 0.86 & & & 0.06 & 0.11 & & & 0.79 & 0.88 & & \\
\hline$\overline{\mathrm{Q} 1}$ & -0.13 & -0.56 & & & -0.33 & -0.84 & & & -0.03 & 0.20 & & & -0.04 & -0.12 & & & -0.38 & -1.01 & & \\
\hline Median & -0.01 & -0.16 & & & -0.09 & 0.17 & & & 0.15 & 0.78 & & & -0.01 & -0.02 & & & 0.14 & -0.10 & & \\
\hline $\mathrm{Q3}$ & 0.41 & 0.62 & & & -0.02 & 0.31 & & & 0.52 & 1.79 & & & 0.01 & 0.04 & & & 0.59 & 0.60 & & \\
\hline Sk & -0.72 & -0.50 & & & -0.85 & -0.04 & & & 1.15 & 0.24 & & & 1.14 & 0.35 & & & 0.37 & 0.09 & & \\
\hline $\mathrm{Ku}$ & 3.13 & 4.38 & & & 3.64 & 2.91 & & & 3.50 & 1.59 & & & 3.51 & 2.19 & & & 2.00 & 2.10 & & \\
\hline & & & & & & & & sponse & eign sto & ck price & to unit & $y U S s h$ & & & & & & & & \\
\hline & & outpu & shock & & & cess liq & dity sho & & & louse pr & ce shocl & & & stock pr & ice shoc & & & fragilit & shock & \\
\hline & & & non $\mathrm{O}$ & CD & & CD & non $\mathrm{OF}$ & & $\mathrm{OE}$ & CD & non $\mathrm{O}$ & CD & & CD & non 0 & ECD & OE & & non $\mathrm{Ol}$ & CD \\
\hline & 2 & 12 & 2 & 12 & 2 & 12 & 2 & 12 & 2 & 12 & 2 & 12 & 2 & 12 & 2 & 12 & 2 & 12 & 2 & 12 \\
\hline Mean & 25.53 & 27.16 & 46.04 & 52.04 & 0.07 & 5.76 & -10.06 & 11.44 & 22.09 & 18.97 & 30.25 & 18.05 & -1.22 & \begin{tabular}{|l|}
-1.61 \\
\end{tabular} & -4.38 & -5.34 & $\begin{array}{l}-9.48 \\
\end{array}$ & -17.76 & 22.77 & -3.00 \\
\hline Std & 28.69 & 35.14 & 50.29 & $\begin{array}{l}64.32 \\
\end{array}$ & 18.17 & 21.55 & 19.30 & 33.84 & 14.93 & 12.78 & 30.14 & 29.66 & 4.33 & 5.39 & 7.75 & 9.43 & 52.08 & 45.65 & 57.54 & 56.73 \\
\hline Q1 & 8.28 & 10.31 & 14.25 & 9.33 & -3.91 & -2.53 & -25.43 & -22.98 & 11.90 & 11.30 & 2.38 & -4.55 & -1.95 & -3.08 & -8.47 & -10.24 & -13.40 & -34.10 & -6.49 & -20.98 \\
\hline Median & 19.10 & 20.20 & 22.15 & 26.65 & 1.44 & 5.21 & $\begin{array}{l}-6.19 \\
\end{array}$ & 5.50 & 17.50 & 15.70 & 23.00 & 9.42 & 0.15 & 0.11 & -5.32 & -5.57 & 4.25 & -4.91 & 14.02 & 0.30 \\
\hline Q3 & 24.30 & 30.60 & $\begin{array}{l}68.78 \\
\end{array}$ & 64.53 & 4.30 & 12.70 & -0.03 & 30.45 & 25.22 & 26.89 & 33.58 & 20.45 & 1.28 & 1.10 & -0.65 & -0.11 & 9.41 & 5.52 & 39.48 & 13.93 \\
\hline Sk & 2.52 & 1.89 & 1.80 & 2.23 & -1.99 & -0.88 & $\begin{array}{c}-0.83 \\
\end{array}$ & 0.67 & 1.27 & 0.74 & 0.67 & 1.07 & -2.25 & -1.80 & -0.38 & -1.07 & $\begin{array}{l}-3.76 \\
\end{array}$ & -1.92 & 0.10 & 0.74 \\
\hline $\mathrm{Ku}$ & 9.68 & 9.06 & 5.95 & 7.89 & 10.40 & 7.52 & 3.13 & 2.65 & 4.15 & 2.95 & 2.25 & 2.83 & 8.14 & 5.96 & 3.46 & 4.79 & 18.79 & 7.40 & 3.42 & 5.05 \\
\hline
\end{tabular}

The Table reports descriptive statistics for the cross section of dynamic responses of selected foreign variables to US shocks: mean (Mean), standard deviation (Std), first (Q1), second (Median) and third (Q3) quartile, index of skewness (Sk) and kurtosis (Ku). Results refers to OECD (+ Israel) and non OECD countries. 
Table 4: Median cumulated response of (selected) foreign variables to US shocks for European and Eastern European countries

\begin{tabular}{|c|c|c|c|c|c|c|c|c|c|c|c|c|c|c|c|c|c|c|c|c|}
\hline \multicolumn{21}{|c|}{ Response of foreign output to unitary US shocks } \\
\hline & \multicolumn{4}{|c|}{ output shock } & \multicolumn{4}{|c|}{ excess liquidity shock } & \multicolumn{4}{|c|}{\begin{tabular}{|c|} 
house price shock \\
\end{tabular}} & \multicolumn{4}{|c|}{ stock price shock } & \multicolumn{4}{|c|}{ fragility shock } \\
\hline & \multicolumn{2}{|c|}{ Europe } & \multicolumn{2}{|c|}{ Eastern Europe } & \multicolumn{2}{|c|}{ Europe } & \multicolumn{2}{|c|}{ Eastern Europe } & \multicolumn{2}{|c|}{ Europe } & \multicolumn{2}{|c|}{ Eastern Europe } & \multicolumn{2}{|c|}{ Europe } & \multicolumn{2}{|c|}{ Eastern Europe } & \multicolumn{2}{|c|}{ Europe } & \multicolumn{2}{|c|}{ Eastern Europe } \\
\hline & 2 & 12 & 2 & 12 & 2 & 12 & 2 & 12 & 2 & 12 & 2 & 12 & 2 & 12 & & 12 & 2 & 12 & & 12 \\
\hline Mean & 0.18 & 0.21 & 0.63 & 0.79 & -0.05 & -0.01 & -0.13 & 0.06 & 0.08 & 0.08 & -0.61 & -0.39 & 0.01 & 0.02 & -0.04 & -0.03 & -0.02 & -0.02 & -1.16 & -0.66 \\
\hline Std & 0.25 & 0.24 & 0.79 & 1.02 & 0.23 & 0.17 & 0.29 & 0.08 & 0.28 & 0.27 & 0.89 & 0.56 & 0.04 & 0.04 & 0.08 & 0.06 & 0.69 & 0.30 & 1.38 & 1.24 \\
\hline Q1 & 0.02 & 0.06 & 0.05 & 0.06 & -0.05 & -0.03 & -0.46 & 0.00 & -0.04 & -0.06 & -1.62 & -1.07 & 0.00 & 0.00 & $\begin{array}{l}-0.11 \\
\end{array}$ & -0.10 & -0.25 & -0.12 & \begin{tabular}{|l|}
-2.73 \\
\end{tabular} & -1.92 \\
\hline Median & 0.10 & 0.15 & 0.12 & 0.15 & -0.01 & 0.03 & 0.00 & 0.01 & 0.01 & 0.02 & -0.34 & -0.25 & 0.00 & 0.01 & -0.02 & -0.01 & \begin{tabular}{|c|}
-0.03 \\
\end{tabular} & -0.03 & -0.89 & -0.32 \\
\hline Q3 & 0.22 & 0.31 & 0.94 & 0.95 & 0.05 & 0.07 & 0.03 & 0.07 & 0.07 & 0.11 & $\begin{array}{l}-0.08 \\
\end{array}$ & 0.02 & 0.01 & 0.01 & 0.00 & -0.01 & 0.02 & 0.02 & -0.36 & 0.00 \\
\hline Sk & 1.42 & 0.76 & 0.51 & 0.77 & -2.27 & -1.70 & -0.99 & 0.70 & 3.02 & 2.60 & -0.93 & -0.60 & 1.90 & 1.96 & -1.07 & -0.48 & 2.26 & 2.03 & -0.75 & -1.14 \\
\hline $\mathrm{Ku}$ & 4.53 & 3.21 & 1.05 & 1.71 & 8.08 & 5.65 & 2.20 & 1.48 & 11.79 & 10.24 & 2.09 & 1.38 & 5.49 & 6.41 & 2.47 & 1.36 & 9.56 & 8.72 & 1.85 & 2.53 \\
\hline \multicolumn{21}{|c|}{ Response of foreign excess credit to unitary US shocks } \\
\hline & & outp & t shock & & & cess lic & iidity sho & & & ouse p & ce shocl & & & stock & ice shock & & & fragilit & shock & \\
\hline & & ope & Eastern & Europe & & & Eastern & urope & & & Eastern & Europe & Eur & & Eastern & Europe & & ope & Eastern & Europe \\
\hline & 2 & 12 & 2 & 12 & 2 & 12 & 2 & 12 & 2 & 12 & 2 & 12 & 2 & 12 & 2 & 12 & 2 & 12 & 2 & 12 \\
\hline Mean & $\begin{array}{l}1.58 \\
\end{array}$ & 2.48 & -2.37 & 0.63 & 0.45 & 1.04 & -0.56 & -2.86 & -0.47 & 0.09 & 1.77 & $\begin{array}{l}-1.38 \\
\end{array}$ & $\begin{array}{l}0.08 \\
\end{array}$ & 0.06 & 0.43 & 0.58 & 2.23 & 1.96 & 5.70 & 2.86 \\
\hline Std & 3.48 & 4.81 & 11.95 & 13.29 & 1.21 & 1.79 & 4.60 & 6.77 & 2.34 & 1.77 & 3.93 & 7.07 & 0.32 & 0.25 & 1.38 & 1.92 & 4.36 & 3.14 & 8.91 & 3.05 \\
\hline Q1 & -0.25 & -0.02 & -13.44 & -12.03 & -0.28 & -0.10 & -5.16 & $\begin{array}{l}-9.92 \\
\end{array}$ & -0.56 & -0.09 & -1.45 & $\begin{array}{l}-8.21 \\
\end{array}$ & -0.07 & -0.09 & -0.47 & -0.66 & -0.01 & 0.09 & -2.25 & -0.39 \\
\hline Median & 0.41 & 0.97 & 0.35 & $\begin{array}{l}2.36 \\
\end{array}$ & 0.16 & 0.52 & 0.05 & -2.80 & -0.06 & 0.15 & 1.07 & $\begin{array}{l}-1.73 \\
\end{array}$ & 0.00 & 0.00 & \begin{tabular}{|c|}
-0.14 \\
\end{tabular} & 0.00 & 0.64 & 0.73 & -0.50 & 3.12 \\
\hline Q3 & 1.51 & 2.28 & 3.91 & 7.50 & 0.39 & 1.79 & 1.64 & 0.41 & 0.21 & 0.56 & 1.60 & 1.41 & 0.03 & 0.04 & 0.28 & 0.22 & 1.84 & 2.43 & 9.76 & 3.73 \\
\hline Sk & 2.83 & 2.64 & -1.27 & -1.13 & 1.85 & 1.10 & -0.94 & -0.70 & -3.10 & -2.61 & 0.96 & -0.64 & 2.66 & 1.69 & 1.08 & 1.13 & 2.29 & 2.11 & 0.39 & -0.05 \\
\hline $\mathrm{Ku}$ & 11.00 & 9.86 & 2.77 & 2.55 & 6.08 & 4.50 & 2.30 & 2.00 & 12.54 & 10.60 & 2.34 & 2.05 & 9.93 & 5.49 & 2.49 & 2.62 & 7.03 & 6.93 & 1.29 & 1.65 \\
\hline & & & & & & & & sponse o & eign ho & se price & to unit & ry US sh & & & & & & & & \\
\hline & 2 & \begin{tabular}{|l|}
12 \\
\end{tabular} & 2 & 12 & 2 & 12 & 2 & 12 & 2 & 12 & 2 & 12 & 2 & 12 & 2 & 12 & 2 & 12 & 2 & 12 \\
\hline Mean & 0.06 & $\begin{array}{l}-0.06 \\
\end{array}$ & & & $\begin{array}{l}-0.19 \\
\end{array}$ & -0.02 & & & 0.52 & 1.08 & & & 0.01 & 0.00 & & & 0.43 & -0.06 & & \\
\hline Std & 0.58 & 1.42 & & & 0.25 & 0.89 & & & 0.72 & 0.86 & & & 0.07 & 0.11 & & & 0.85 & 0.91 & & \\
\hline Q1 & -0.14 & -0.52 & & & -0.37 & -0.79 & & & -0.01 & 0.48 & & & -0.04 & -0.10 & & & -0.33 & $\begin{array}{l}-0.88 \\
\end{array}$ & & \\
\hline Median & -0.01 & -0.22 & & & -0.10 & 0.14 & & & 0.33 & 0.81 & & & -0.01 & -0.01 & & & 0.34 & -0.21 & & \\
\hline Q3 & 0.38 & 0.61 & & & -0.05 & 0.33 & & & 0.81 & 1.81 & & & 0.02 & 0.08 & & & 0.98 & 0.50 & & \\
\hline Sk & -0.68 & -0.43 & & & -1.21 & -0.02 & & & 0.85 & 0.05 & & & 1.01 & 0.24 & & & 0.12 & 0.18 & & \\
\hline $\mathrm{Ku}$ & 2.75 & 3.64 & & & 3.63 & 2.86 & & & 2.76 & 1.45 & & & 2.88 & 2.10 & & & 1.71 & 2.10 & & \\
\hline & & & & & & & & sponse & reign sto & k price & to unitc & $y U S s h$ & & & & & & & & \\
\hline & & outp & t shock & & & cess lic & lidity sho & & & ouse p & ce shocl & & & stock ${ }_{F}$ & ice shock & & & fragilit & shock & \\
\hline & & ope & Eastern & Europe & & & Eastern & urope & & & Eastern & Europe & Eur & & Eastern & Europe & & ope & Eastern & Europe \\
\hline & 2 & \begin{tabular}{|l|}
12 \\
\end{tabular} & 2 & 12 & 2 & 12 & 2 & 12 & 2 & 12 & 2 & 12 & 2 & 12 & 2 & 12 & 2 & 12 & 2 & 12 \\
\hline Mean & 25.21 & 26.77 & 47.33 & 47.11 & 4.05 & $\begin{array}{l}7.36 \\
\end{array}$ & 2.85 & $\begin{array}{r}6.48 \\
\end{array}$ & 21.27 & 18.97 & 32.19 & 25.42 & -0.17 & -0.10 & -4.50 & -6.66 & $\begin{array}{l}-7.87 \\
\end{array}$ & -12.07 & -10.21 & -43.16 \\
\hline Std & 22.84 & 19.81 & 77.25 & 112.31 & 7.77 & 9.76 & 22.29 & 21.88 & 13.50 & 11.78 & 33.54 & 24.63 & 2.27 & 2.21 & 9.58 & 13.48 & 65.11 & 46.55 & 47.69 & 40.20 \\
\hline Q1 & 9.87 & 11.43 & 8.07 & -20.10 & -2.14 & 0.96 & -19.75 & -18.95 & 10.93 & 8.03 & 6.90 & 6.64 & -1.73 & -1.66 & -13.60 & -19.78 & -0.43 & -12.28 & -45.40 & -87.25 \\
\hline Median & 19.55 & 21.45 & 19.90 & 11.53 & 3.15 & 7.30 & -1.21 & 3.10 & 16.93 & 15.95 & 19.52 & 12.80 & 0.48 & 0.56 & -1.74 & -3.52 & 6.41 & -3.58 & -26.20 & -55.50 \\
\hline Q3 & 25.63 & 32.10 & 21.98 & 20.65 & 5.11 & 12.18 & 12.96 & 21.10 & 24.23 & 27.17 & 33.42 & 28.96 & 1.20 & 1.14 & 0.32 & -1.58 & 10.50 & 4.66 & -10.98 & -17.75 \\
\hline Sk & 1.68 & 1.37 & 1.32 & 1.18 & 1.42 & 0.45 & 0.23 & -0.21 & 1.33 & 0.39 & 0.81 & 0.72 & -1.23 & -1.07 & -1.25 & -1.04 & -3.28 & -2.97 & 0.83 & $\begin{array}{l}-0.03 \\
\end{array}$ \\
\hline $\mathrm{Ku}$ & 4.82 & 4.71 & 2.86 & 2.68 & 5.08 & 3.70 & 1.07 & 1.05 & 4.10 & 2.09 & 1.98 & 1.76 & 3.97 & 3.16 & 2.74 & 2.48 & 13.24 & 11.82 & 2.14 & 1.03 \\
\hline
\end{tabular}

The Table reports descriptive statistics for the cross section of dynamic responses of selected foreign variables to US shocks: mean (Mean), standard deviation (Std), first (Q1), second (Median) and third (Q3) quartile, index of skewness (Sk) and kurtosis (Ku). Results refers to European (+ Canada) and Eastern European (+ Russia) countries. 
Table 5: Median cumulated response of (selected) foreign variables to US shocks for Asian and Latin American countries

\begin{tabular}{|c|c|c|c|c|c|c|c|c|c|c|c|c|c|c|c|c|c|c|c|c|}
\hline \multicolumn{21}{|c|}{ Response of foreign output to unitary US shocks } \\
\hline & \multicolumn{4}{|c|}{ output shock } & \multicolumn{4}{|c|}{ excess liquidity shock } & \multicolumn{4}{|c|}{ house price shock } & \multicolumn{4}{|c|}{ stock price shock } & \multicolumn{4}{|c|}{ fragility shock } \\
\hline & \multicolumn{2}{|c|}{ Asia } & \multicolumn{2}{|c|}{ Latin America } & \multicolumn{2}{|c|}{ Asia } & \multicolumn{2}{|c|}{ Latin America } & \multicolumn{2}{|c|}{ Asia } & \multicolumn{2}{|c|}{ Latin America } & \multicolumn{2}{|c|}{ Asia } & \multicolumn{2}{|c|}{ Latin America } & \multicolumn{2}{|c|}{ Asia } & \multicolumn{2}{|c|}{ Latin America } \\
\hline & 2 & 12 & 2 & 12 & 2 & 12 & & 12 & 2 & 12 & & 12 & 2 & 12 & & 12 & 2 & 12 & & \\
\hline Mean & 0.94 & 1.46 & 0.42 & 0.67 & 0.13 & 0.52 & 0.06 & 0.02 & 0.05 & -0.07 & -0.11 & -0.40 & 0.06 & 0.00 & 0.01 & 0.02 & -0.60 & -0.90 & -0.17 & -0.05 \\
\hline Std & 1.79 & 2.86 & 0.90 & 1.50 & 2.17 & 2.18 & 0.49 & 0.75 & 1.91 & 2.15 & 0.62 & 0.52 & 0.28 & 0.24 & 0.04 & 0.05 & 8.62 & 8.79 & 1.02 & 1.09 \\
\hline Q1 & 0.17 & 0.15 & -0.39 & -0.67 & -0.44 & -0.49 & -0.42 & -0.74 & -0.10 & -0.46 & -0.66 & -0.89 & -0.06 & -0.06 & -0.03 & -0.03 & -0.47 & -0.75 & -1.04 & -0.80 \\
\hline Median & 0.32 & 0.33 & 0.38 & 0.66 & -0.04 & -0.04 & -0.06 & -0.20 & 0.04 & 0.00 & -0.21 & -0.30 & -0.01 & -0.03 & -0.01 & 0.00 & -0.03 & -0.10 & -0.56 & -0.62 \\
\hline Q3 & 0.90 & 1.25 & 0.57 & 0.73 & 0.47 & 0.64 & 0.32 & 0.41 & 0.36 & 0.24 & 0.00 & -0.14 & 0.10 & 0.02 & 0.01 & 0.04 & 0.60 & 0.33 & 0.06 & 0.00 \\
\hline Sk & 2.07 & 2.49 & -0.09 & 0.37 & -0.85 & 0.53 & -0.17 & -0.08 & -0.26 & 0.01 & -0.01 & -1.24 & 0.91 & -0.73 & 0.85 & 0.41 & -1.28 & -1.05 & 0.53 & 0.88 \\
\hline $\mathrm{Ku}$ & 7.11 & 8.56 & 1.95 & 2.04 & 4.83 & 2.57 & 1.40 & 1.32 & 3.50 & 4.05 & 1.92 & 2.72 & 4.56 & 4.82 & 2.08 & 1.53 & 7.09 & 6.75 & 1.42 & 1.94 \\
\hline \multicolumn{21}{|c|}{ Response of foreign excess credit to unitary US shocks } \\
\hline & \multicolumn{4}{|c|}{ output shock } & \multicolumn{4}{|c|}{ excess liquidity shock } & \multicolumn{4}{|c|}{\begin{tabular}{|l|} 
house price shock \\
\end{tabular}} & \multicolumn{4}{|c|}{ stock price shock } & \multicolumn{4}{|c|}{ fragility shock } \\
\hline & & ia & Latin & merica & A & & Latin $\mathrm{A}$ & merica & & & Latin 1 & merica & $\mathbf{A}$ & & Latin $A$ & merica & & & Latin A & merica \\
\hline & 2 & 12 & 2 & 12 & 2 & 12 & 2 & 12 & 2 & 12 & 2 & 12 & 2 & 12 & 2 & 12 & 2 & 12 & 2 & 12 \\
\hline Mean & 2.45 & 3.67 & -0.72 & 1.11 & -0.42 & -1.73 & 0.65 & -2.14 & -1.29 & -1.26 & 0.70 & 0.33 & -0.75 & -0.72 & 0.46 & 0.52 & 0.93 & 2.39 & 2.76 & 2.27 \\
\hline Std & 8.65 & 14.64 & 7.09 & 10.26 & 6.77 & 8.04 & 1.98 & 5.48 & 7.24 & 7.36 & 2.05 & 2.75 & 1.77 & 1.57 & 0.53 & 0.53 & 16.44 & 14.08 & 7.93 & 10.15 \\
\hline Q1 & -1.45 & -2.38 & -6.77 & -7.82 & -1.83 & -2.45 & -1.01 & -8.44 & -1.03 & -0.79 & -1.56 & -2.84 & -0.80 & -0.68 & 0.07 & 0.02 & -6.69 & -2.83 & -5.20 & -7.14 \\
\hline Median & -0.45 & -0.45 & -1.80 & -1.65 & -0.16 & -0.21 & 0.37 & -1.11 & -0.11 & -0.24 & 0.92 & 0.86 & -0.11 & -0.11 & 0.10 & 0.26 & -0.29 & 0.27 & -0.02 & -0.93 \\
\hline Q3 & 1.17 & 0.85 & -0.36 & 2.52 & 1.70 & 1.18 & 0.59 & 0.89 & 0.31 & 0.77 & 1.67 & 1.50 & -0.02 & -0.03 & 0.62 & 0.83 & 2.74 & 3.13 & 6.85 & 6.21 \\
\hline Sk & 2.11 & 2.50 & 0.50 & 0.64 & -1.97 & -2.10 & 0.92 & -0.67 & -2.77 & -2.82 & -0.58 & -0.30 & -2.36 & -1.86 & 0.57 & 0.18 & 1.40 & 1.12 & -0.07 & 0.13 \\
\hline $\mathrm{Ku}$ & 6.86 & 8.66 & 2.09 & 1.76 & 7.44 & 7.46 & 2.28 & 1.73 & 10.03 & 10.20 & 1.80 & 1.43 & 7.86 & 5.22 & 1.23 & 1.07 & 6.26 & 5.71 & 1.35 & 1.33 \\
\hline & & & & & & & & esponse & reign st & k price & to unit & $y U S s h$ & & & & & & & & \\
\hline & & outpt & t shock & & & cess liqu & dity shoc & & & louse $p$ & ice shoc & & & stock $p$ & ice shocl & & & fragili & y shock & \\
\hline & & ia & Latin & merica & & & Latin A & merica & & & Latin & merica & A & & Latin $A$ & merica & & & Latin A & merica \\
\hline & 2 & 12 & 2 & 12 & 2 & 12 & 2 & 12 & 2 & 12 & 2 & 12 & 2 & 12 & 2 & 12 & 2 & 12 & 2 & 12 \\
\hline Mean & 34.68 & 38.14 & 43.28 & 47.82 & -15.30 & 4.65 & -7.68 & 14.53 & 24.02 & 17.00 & 36.07 & 17.71 & 0.93 & 2.39 & -6.49 & -7.37 & 28.52 & 6.53 & -18.03 & -30.92 \\
\hline Std & 38.43 & 43.17 & 37.20 & 40.04 & 23.75 & 41.81 & 18.09 & 25.90 & 23.71 & 25.41 & 25.84 & 27.54 & 16.44 & 14.08 & 5.34 & 5.87 & 45.81 & 54.47 & 48.83 & 52.68 \\
\hline Q1 & 6.83 & 3.85 & 13.55 & 18.00 & -28.43 & -24.13 & -27.00 & -13.87 & 8.34 & -5.72 & 11.67 & -0.14 & -6.69 & -2.83 & -11.27 & -12.35 & -5.83 & -20.18 & -73.55 & -94.80 \\
\hline Median & 19.75 & 25.20 & 18.20 & 26.00 & -8.32 & -0.94 & -7.99 & 6.58 & 20.40 & 13.00 & 23.80 & 7.50 & $\begin{array}{l}-0.29 \\
\end{array}$ & 0.27 & -8.74 & -10.70 & 11.57 & 5.13 & 4.82 & -20.00 \\
\hline Q3 & 39.45 & 58.00 & 55.20 & 55.45 & 0.67 & 11.19 & -0.41 & 34.05 & 28.78 & 22.53 & 50.85 & 13.25 & 2.74 & 3.13 & -3.85 & -4.46 & 34.83 & 18.10 & 6.91 & 2.26 \\
\hline Sk & 1.46 & 1.63 & 0.57 & 0.66 & -1.39 & 0.43 & -0.27 & -0.35 & 0.91 & 1.11 & 0.27 & 1.21 & 1.40 & 1.12 & 0.24 & 0.43 & 1.47 & 0.97 & -0.95 & -0.54 \\
\hline $\mathrm{Ku}$ & 4.37 & 5.48 & 1.42 & 1.45 & 3.73 & 2.62 & 1.66 & 1.22 & 3.26 & 3.86 & 1.04 & 2.68 & 6.26 & 5.71 & 1.13 & 1.03 & 3.91 & 4.93 & 2.11 & 1.36 \\
\hline
\end{tabular}

The Table reports descriptive statistics for the cross section of dynamic responses of selected foreign variables to US shocks: mean (Mean), standard deviation (Std), first (Q1), second (Median) and third (Q3) quartile, index of skewness (Sk) and kurtosis (Ku). Results refers to Asian and Latin American countries. 
APPENDIX A: Robustness analysis

Table 1A: Median cumulated impulse response analysis for US variables (selected shocks)

\begin{tabular}{|c|c|c|c|c|c|c|c|c|c|c|c|c|c|c|c|}
\hline $\operatorname{Sh} \downarrow$ & Resp $\rightarrow$ & $e$ & $g$ & $p d$ & $c$ & $i$ & cad & $\pi$ & exl & $s$ & $l$ & $h$ & $e r$ & $f$ & $f r$ \\
\hline & 2 & 0.25 & 0.11 & -0.07 & 0.08 & 0.12 & 0.03 & -0.04 & 0.03 & 0.05 & -0.07 & 0.06 & 0.25 & -0.14 & -0.02 \\
\hline \multirow[t]{2}{*}{$e$} & 4 & 0.25 & 0.12 & -0.02 & 0.06 & 0.28 & 0.02 & 0.01 & 0.06 & 0.10 & 0.01 & -0.03 & 0.20 & -0.13 & 0.00 \\
\hline & 12 & 0.19 & 0.02 & 0.00 & 0.03 & -0.07 & -0.01 & 0.03 & 0.17 & 0.02 & 0.01 & -0.13 & 0.23 & -0.21 & 0.00 \\
\hline & & & & & & & & & & & & & & & \\
\hline & 2 & 0.12 & 0.33 & 0.06 & 0.06 & 0.26 & 0.07 & -0.12 & -0.27 & 0.10 & 0.23 & 0.02 & -0.46 & 0.05 & -0.04 \\
\hline \multirow[t]{2}{*}{$g$} & 4 & 0.15 & 0.42 & 0.00 & 0.01 & 0.33 & 0.07 & -0.19 & -0.20 & 0.02 & 0.09 & 0.11 & -0.20 & -0.46 & -0.02 \\
\hline & 12 & 0.19 & 0.44 & 0.00 & -0.01 & 0.35 & 0.05 & -0.29 & -0.12 & -0.03 & -0.04 & 0.32 & -0.65 & 0.18 & -0.02 \\
\hline & & & & & & & & & & & & & & & \\
\hline & 2 & -0.01 & 0.04 & 0.05 & -0.03 & -0.04 & -0.06 & -0.01 & -0.09 & 0.24 & 0.38 & -0.09 & 0.16 & -0.80 & 0.02 \\
\hline \multirow[t]{2}{*}{$p d$} & 4 & 0.01 & 0.10 & 0.01 & -0.01 & 0.07 & -0.05 & -0.06 & -0.15 & 0.09 & 0.15 & -0.01 & -0.02 & -0.33 & -0.05 \\
\hline & 12 & 0.09 & 0.24 & 0.00 & 0.00 & 0.38 & 0.02 & -0.14 & -0.25 & -0.02 & -0.01 & 0.14 & -0.15 & 0.58 & -0.03 \\
\hline & & & & & & & & & & & & & & & \\
\hline & 2 & 0.02 & -0.01 & 0.07 & -0.08 & -0.08 & 0.26 & -0.12 & 0.08 & 0.01 & 0.09 & -0.11 & -0.13 & -2.07 & 0.00 \\
\hline \multirow[t]{2}{*}{ cad } & 4 & -0.01 & -0.07 & 0.05 & -0.19 & -0.42 & 0.19 & -0.23 & 0.26 & -0.05 & 0.00 & -0.08 & -0.20 & -2.69 & 0.01 \\
\hline & 12 & -0.04 & -0.16 & 0.00 & -0.32 & -0.79 & 0.04 & -0.30 & 0.40 & -0.03 & -0.05 & 0.10 & -0.87 & -2.52 & -0.01 \\
\hline & & & & & & & & & & & & & & & \\
\hline & 2 & -0.02 & -0.17 & 0.04 & -0.06 & -0.13 & -0.04 & 0.46 & -0.05 & -0.18 & -0.18 & -0.44 & -0.09 & -1.58 & 0.15 \\
\hline \multirow[t]{2}{*}{$\pi$} & 4 & -0.11 & -0.24 & 0.02 & -0.01 & -0.21 & -0.07 & 0.52 & -0.02 & 0.10 & 0.06 & -0.45 & 0.07 & -1.79 & 0.11 \\
\hline & 12 & -0.13 & -0.25 & 0.00 & 0.13 & -0.25 & -0.01 & 0.59 & 0.08 & 0.08 & 0.08 & -0.54 & 0.47 & -1.67 & 0.03 \\
\hline & & & & & & & & & & & & & & & \\
\hline & 2 & 0.02 & 0.01 & -0.05 & 0.01 & -0.06 & 0.01 & 0.03 & 0.40 & -0.18 & -0.14 & -0.06 & 0.02 & -0.41 & 0.03 \\
\hline \multirow[t]{2}{*}{ exl } & 4 & 0.04 & 0.09 & 0.02 & 0.04 & 0.19 & 0.02 & 0.03 & $\mathbf{0 . 3 7}$ & 0.07 & 0.07 & -0.12 & -0.07 & 0.29 & 0.02 \\
\hline & 12 & 0.06 & 0.10 & 0.00 & 0.04 & 0.15 & 0.01 & -0.02 & 0.45 & 0.01 & 0.00 & -0.03 & -0.06 & 0.29 & 0.00 \\
\hline & 2 & -0.02 & -0.06 & -0.06 & -0.06 & -0.34 & -0.07 & 0.09 & 0.10 & 0.18 & -0.15 & -0.14 & 0.35 & -0.98 & 0.11 \\
\hline \multirow[t]{2}{*}{$s$} & 4 & -0.11 & -0.26 & -0.08 & -0.03 & -0.68 & -0.12 & 0.21 & 0.34 & 0.18 & 0.01 & -0.24 & 0.44 & -0.48 & 0.11 \\
\hline & 12 & -0.21 & -0.33 & 0.00 & 0.04 & -0.97 & -0.07 & $\begin{array}{l}0.31 \\
\end{array}$ & $\begin{array}{l}0.38 \\
\end{array}$ & 0.07 & 0.09 & -0.63 & 0.88 & -0.65 & 0.03 \\
\hline & 2 & 0.02 & 0.04 & -0.09 & 0.11 & 0.15 & 0.02 & 0.06 & -0.05 & -0.18 & -0.24 & 0.62 & -0.17 & 1.09 & -0.04 \\
\hline \multirow[t]{2}{*}{$\boldsymbol{h}$} & 4 & 0.08 & 0.10 & -0.05 & 0.20 & 0.61 & 0.09 & 0.12 & -0.13 & 0.07 & -0.05 & 0.80 & -0.30 & 1.28 & -0.02 \\
\hline & 12 & 0.03 & -0.07 & 0.00 & 0.19 & 0.23 & 0.03 & 0.19 & 0.05 & 0.02 & -0.02 & 0.88 & -0.34 & 0.65 & 0.02 \\
\hline & & & & & & & & & & & & & & & \\
\hline & 2 & 0.01 & 0.04 & 0.06 & -0.02 & 0.18 & 0.09 & -0.03 & -0.06 & 0.02 & 0.08 & 0.02 & 0.24 & 3.57 & -0.03 \\
\hline \multirow[t]{3}{*}{$f$} & 4 & 0.02 & 0.08 & 0.01 & 0.00 & 0.11 & 0.08 & -0.08 & -0.03 & -0.02 & -0.01 & 0.16 & -0.10 & 3.77 & -0.05 \\
\hline & 12 & 0.05 & 0.07 & 0.00 & -0.07 & 0.12 & 0.03 & -0.14 & 0.01 & -0.02 & -0.04 & 0.29 & -0.39 & 3.92 & -0.01 \\
\hline & 2 & -0.03 & -0.05 & 0.03 & 0.07 & -0.18 & 0.03 & -0.02 & 0.01 & 0.04 & 0.07 & 0.06 & 0.03 & 0.32 & 0.16 \\
\hline \multirow[t]{2}{*}{$f r$} & 4 & 0.01 & 0.05 & 0.00 & 0.15 & 0.12 & 0.06 & -0.03 & 0.00 & 0.06 & -0.01 & 0.16 & -0.01 & 0.33 & 0.08 \\
\hline & 12 & -0.02 & -0.05 & 0.01 & 0.20 & -0.16 & 0.02 & 0.01 & 0.27 & 0.05 & 0.03 & 0.19 & 0.13 & -0.19 & 0.03 \\
\hline
\end{tabular}

The Table reports the results of the median cumulated impulse response analysis for the US variables (columns), relative to the various shocks (rows). The variables are real GDP $(g)$, civilian employment $(e)$, real private consumption $(c)$, real private investment $(i)$, fiscal deficit to GDP $(p d)$, current account deficit to GDP $(c a d)$, CPI all items index $(\pi)$, three-month Treasury Bills real rate $(s)$, 10-year Federal government securities real rate $(l)$, real house prices $(h)$, real share prices $(f)$, the economic/financial fragility index $(f r)$, the excess liquidity index $($ exl). Figures in bold are significant at the $10 \%$ level. 
DEPARTMENT OF ECONOMICS AND PUBLIC FINANCE "G. PRATO" UNIVERSITY OF TORINO

Corso Unione Sovietica 218 bis - 10134 Torino (ITALY)

Phone: +390116706128 - Fax: +390116706062

Web page: http://eco83.econ.unito.it/prato/ 